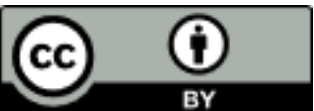

\section{Ivana Šarić Šokčević}

Fakultät für Geistes- und Sozialwissen-

schaften in Osijek
UDK 811.112.2'367.625'232=112.2

Origineller Foschungsartikel

Angenommen für Publikation am 26.05.2021.

https://doi.org/10.29162/jez.2021.4

\title{
Die Beziehung zwischen der Bewusstheit und den Lehrmethoden beim Erlernen der (nicht) passivfähigen Verben im Deutschen als Fremd- sprache
}

Das Hauptziel dieser Untersuchung war es, die Beziehung zwischen der Bewusstheit (engl. awareness) und den expliziten und impliziten Lehrmethoden bezüglich des Erlernens einer komplexen, mehrdimensionalen fremdsprachlichen Zielstruktur, der (nicht) passivfähigen Verben im Deutschen als Fremdsprache, zu erforschen. Viele Untersuchungen aus dem Bereich des Zweit- und Fremdsprachenerwerbs (z. B. Schmidt 1990; Robinson 1995; Leow 2001) haben bis jetzt Schmidts Noticing-Hypothese (1990; 1993 ; 1995; 2001) bestätigt, in der davon ausgegangen wird, dass die Aufmerksamkeit eine Schlüsselrolle beim erfolgreichen Erlernen zielsprachlicher Strukturen spielt, da es ohne Aufmerksamkeit nicht zum Bemerken der Zielstruktur im Input kommen kann, ohne das Bemerken nicht zum Erlernen. Es wird somit nicht nur der Aufmerksamkeit, sondern auch der Bewusstheit eine ausschlaggebende Rolle zugesprochen. Schmidt geht in seiner Noticing-Hypothese $(1990 ; 1993 ; 1995 ; 2001)$ von der niedrigeren Bewusstheitsebene, der Ebene des Bemerkens, und der höheren Bewusstheitsebene, der Ebene des Verstehens, aus. Das Verstehen des sprachlichen Inputs kann auf zwei verschiedene Weisen zustande kommen: durch implizites und/oder explizites Lernen/Lehrmethoden (DeKeyser 2003; Ellis, R. 2005). Um diese Beziehungen detaillierter zu beleuchten, wurde in der vorliegenden Untersuchung ein Experiment in drei Phasen durchgeführt. Die Teilnehmer waren kroatische Gymnasiasten, die Deutsch als Fremdsprache lernen, und Germanistikstudenten eines Masterstudiums. Zur Datenerhebung bezüglich der Bewusstheitsebenen wurde die Think-aloud-Methode angewandt. Die erhaltenen Daten wurden qualitativ und quantitativ mit einer statistischen Analyse (mehrfaktorielle Varianzanalyse) bearbeitet. Die Re- 


\section{Ivana Šarić Šokčević: \\ Die Beziehung zwischen der Bewusstheit und den Lehrmethoden beim Er- lernen der (nicht) passivfähigen Verben im Deutschen als Fremdsprache}

sultate zeigen bezüglich der Erforschung der Rolle der Bewusstheit in der Input-intake-Phase eine starke positive Beziehung zwischen den Lernergebnissen der expliziten Lehrmethode und der höheren Bewusstheitsebene, aber auch der impliziten Lehrmethode und der niedrigeren Bewusstheitsebene. Die Lernergebnisse der Untersuchungsteilnehmer, die der expliziten Lehrmethode ausgesetzt waren, haben, insgesamt betrachtet, bessere Resultate gezeigt. Es wurde auch eine Interaktion zwischen den Untersuchungsteilnehmern, die sich auf einem höheren Sprachkenntnisstand befinden, und der niedrigeren Bewusstheitsebene festgestellt, wobei diese Untersuchungsteilnehmer bessere Lernleistungen beim unmittelbaren Posttest zeigten. Die erhaltenen Daten bestätigen die Annahmen von Schmidts Noticing-Hypothese (1990) und die Untersuchungsergebnisse ähnlicher Untersuchungen (z. B. Leow 1997; 2001; Medina 2015).

Schlüsselwörter: Zweit- und Fremdsprachenerwerbsprozess; Input-intakePhase; (nicht) passivfähige Verben im Deutschen als Fremdsprache; Bewusstheit; Bewusstheitsebenen; explizite und implizite Lehrmethode.

\section{Einleitung}

Heute sind sich viele Zweit- und Fremdsprachenforscher (z. B. Schmidt 1990; Robinson 1995; Leow 2001) darüber einig, dass der Lernprozess aus verschiedenen Mikro- und Makroprozessen besteht, die Lernende beim Erlernen einer Zielstruktur durchlaufen. In diesen komplexen Prozessen scheint die Aufmerksamkeit (engl. attention) eine wichtige Rolle zu spielen. Es konnte in Untersuchungen aus dem Bereich des Zweit- und Fremdsprachenerwerbs (z. B. Schmidt 1990; Robinson 1995; Leow 2001) bestätigt werden, dass es zum effektiveren Erlernen einer Zielstruktur kommt, wenn die Aufmerksamkeit bewusst auf bestimmte Stimuli im Input gelenkt wird und diese dann in den restlichen Phasen des fremdsprachlichen Lernprozesses verarbeitet werden. Es wird somit angenommen, dass die Aufmerksamkeit eine wichtige Grundlage für einen effektiven Lernprozess bietet. Lernende bearbeiten durch einen aufmerksamen und kontrollierten Zugriff die dargebotenen Informationen und stellen diese dann für die weiteren Phasen des Lernprozesses zur Verfügung. Einige Zweit- und Fremdsprachenforscher (Schmidt 1990; Robinson 1995; Leow 1997) haben sich der Erforschung der unterschiedlichen Lernphasen gewidmet und dabei festgestellt, dass die Aufmerksamkeit besonders in der frühen Phase des Lernprozesses, der Input-Intake-Phase, die ausschlaggebende Rolle spielt. Für solche Annahmen finden sie theoretischen Halt in Schmidts NoticingHypothese (1990; 1993; 1995; 2001). Schmidt (ebd.) nimmt an, dass die Aufmerksamkeit eine Schlüsselrolle beim erfolgreichen Erlernen fremdsprachlicher Ziel- 


\section{JEZIKOSLOVLJE \\ 22.1 (2021): 99-148}

strukturen spielt. Schmidt (ebd.) geht davon aus, dass das Erlernen fremdsprachlicher Zielstrukturen immer mit einem gewissen Grad an Aufmerksamkeit verbunden ist, wobei es ohne Aufmerksamkeit nicht zum Bemerken (engl. noticing) der fremdsprachlichen Zielstruktur im Input kommen kann. Ferner kann ohne das Bemerken auch nicht der Verarbeitungsprozess, der zum Erlernen der Zielstruktur führt, gestartet werden. Das Verstehen (engl. understanding) dieses bemerkten Materials im Input ist der nächste Schritt, der in diesem Prozess gewährleistet sein muss, wobei mehr Bemerken und Verstehen zu besseren Lernleistungen führen. Schmidt (ebd.) und andere Zweit- und Fremdsprachenforscher (Rosa und O'Neill 1999; Leow 2000, 2001; Bagarić 2001; Rosa und Leow 2004; de la Fuente 2015; Medina 2015) nehmen an, dass es Strukturen gibt, die zum Erlernen einen höheren, andere hingegen einen niedrigeren Grad an Aufmerksamkeit fordern. Es wird daher nicht nur der Aufmerksamkeit eine wichtige Rolle im Lernprozess zugeteilt erteilt, sondern auch der Bewusstheit (engl. awareness), da Schmidt (1995: 1) annimmt, dass die Aufmerksamkeit „fast isomorph mit der Bewusstheit ist“". Schmidt (1990; 1993; 1995; 2001; 2010) hat sich nicht nur mit der Rolle des Bewusstseins und den jeweiligen Bedeutungen auseinandergesetzt, sondern auch mit den damit verbundenen Bewusstheitsebenen, die im Lernprozess eine Rolle zu spielen scheinen. Schmidt (ebd.) erläutert in seinen theoretischen Auslegungen, dass für das Erlernen einer Zielstruktur zwei verschiedene Bewusstheitsebenen eine Rolle spielen: die Noticing-Ebene und die Understanding-Ebene. Um den Lernprozess zu starten, muss vom Lernenden die niedrigere Bewusstheitsebene, die noticing-Ebene, erreicht werden, d. h. die fremdsprachliche Zielstruktur muss vom Lernenden zuerst im Input bemerkt werden. In diesem Prozess spielt aber auch das Verstehen, die höhere Bewusstheitsebene, die Understanding-Ebene, eine wichtige Rolle. Diese höhere Bewusstheitsebene, d. h. das Verstehen des bemerkten Inputs, dient dann weiter einem differenzierten Verständnis der zugrunde liegenden fremdsprachlichen Zielstruktur. Das Verstehen der Zielstruktur kann auf zwei verschiedene Weisen erfolgen: durch implizites und/oder explizites Lernen oder implizite und/oder explizite Lehrmethoden (DeKeyser 2003; Ellis, R. 2005; Leow 2015). In den letzten Jahrzehnten hat man sich maßgebend mit der Wirksamkeit der expliziten und impliziten Instruktionsverfahren in den verschiedenen Phasen des Lernprozesses beschäftigt (Fotos 1993; Leow 1998; Radwan 2005; Andrews 2007). Dabei wurden aber auch einige zusätzliche Merkmale der Lernenden thematisiert, wie beispielsweise der Sprachkenntnisstand (engl. level of proficiency) der Lernenden (niedrigerer Wissensstand vs. höherer Wissensstand) (z. B. Gass, Svetics und Lemelin 2003; Calderón 2013) oder auch die Komplexität der Zielstruktur (einfache vs. komplexe Zielstrukturen) (z. B. Robinson 1996; DeKeyser 2003). Es besteht auch 
Ivana Šarić Šokčević:

Die Beziehung zwischen der Bewusstheit und den Lehrmethoden beim Erlernen der (nicht) passivfähigen Verben im Deutschen als Fremdsprache

weiterhin ein Bedarf an empirischen Untersuchungen bezüglich der Beziehung der Bewusstheit (Schmidt 1990; Tomlin und Villa 1994; Robinson 1995; Leow 1997), der Bewusstheitsebenen (z. B. Leow 1997; Faretta-Stutenberg und Morgan-Short 2011; Chan und Leung 2014; Medina 2015) und der expliziten und impliziten Lehrmethoden (z. B. Alanen 1995; Robinson 1996, 1997; Radwan 2005).

\section{Theoretischer Hintergrund}

\subsection{Die Phasen des fremdsprachlichen Lernprozesses}

Zweit- und Fremdsprachenforscher scheinen heute einvernehmlich der Meinung zu sein, dass das Erlernen einer Zweit- oder Fremdsprache die folgenden Phasen durchläuft: ,Input > Intake ${ }^{1}>$ Internal system > Output“ (Leow 2015: 17). Daraus geht hervor, dass der fremdsprachliche Lernprozess gestartet wird, wenn Lernende zielsprachlichem Material (Input) ausgesetzt werden. Nur Teile dieses Inputs werden tatsächlich weiter in Intake verwandelt, welches dann für weitere Verarbeitungsprozesse verfügbar gemacht wird. Diese Verarbeitungsprozesse dienen wiederum dazu, das Lernmaterial, das als Intake aufgenommen wurde, am Ende des fremdsprachlichen Lernprozesses zu Output, dem Endprodukt, das vom Lernenden sprachlich erfasst, verstanden und produziert werden kann, zu verarbeiten. In der Diskussion bezüglich dieser verschiedenen Lernphasen und ihrer Wichtigkeit im fremdsprachlichen Lernprozess taucht immer wieder die frühe Phase, die Inputintake-Phase, sowohl als äußerst relevante, aber zugleich auch diskutable Schnittstelle auf. Leow (2015: 17) stellt in der unten dargebotenen Abbildung seine detaillierten Ansichten und Unterscheidungen bezüglich der verschiedenen Phasen, die sich im fremdsprachlichen Lernprozess vollziehen, wie folgt dar:

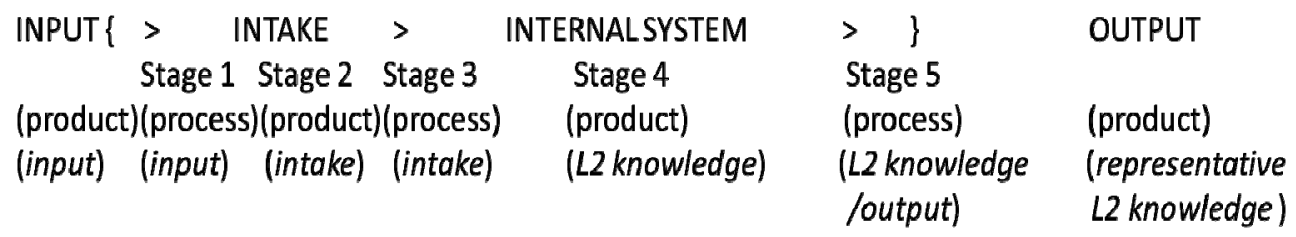

Abbildung 1. Der Lernprozess im Zweit- und Fremdsprachenerwerb nach Leow (2015: 17)

\footnotetext{
${ }^{1}$ Intake wird wie folgt definiert: „(...) that part of the language to which learners are exposed, that actually "goes in" and plays a role in language learning" (Richard und Schmidt 2002: 262).
} 
Anhand der Abbildung kann geschlussfolgert werden, dass Leow (2015) einen entscheidenden Unterschied zwischen den jeweiligen Prozessen im Lernprozess macht, die sich während der jeweiligen Lernphasen abspielen, und den entsprechenden Produkten, die nach der Vollziehung des jeweiligen Lernprozesses, entstehen. Über die verschiedenen Prozesse, Produkte und besonders Faktoren, die bei diesen frühen Phasen im fremdsprachlichen Lernprozess die ausschlaggebende Rolle spielen, gibt es jedoch bis heute keinen eindeutigen Einklang unter Zweitund Fremdsprachenforschern (Schmidt 1990, 1993, 1995, 2001, 2010; Tomlin und Villa 1994; Robinson 1995; VanPatten 1996; Gass 1998; Leow 2015). Umstritten bleibt auch weiterhin die Rolle der Aufmerksamkeit (engl. attention) und der Bewusstheit (engl. awareness).

\subsection{Zu den Bewusstheitsebenen im Lernprozess}

Zwei Jahrzehnte nach der Veröffentlichung der theoretischen Auslegungen bezüglich der Noticing-Hypothese hebt selbst Schmidt (2010) weiterhin hervor, das Bewusstsein als Bewusstheit (engl. consciousness as awareness) spiele noch immer eine wichtige, wenn auch teilweise kontroverse Rolle im Kontext des Zweitsprach- und Fremdsprachenlernens. Genau deshalb haben sich sowohl Kognitionspsychologen und -wissenschaftler (z. B. Posner 1992) als auch Zweit- und Fremdsprachenwissenschaftler (z. B. Leow 1997, 2000, 2001; Rosa und O'Neill 1999; Bagarić 2001; Rosa und Leow 2004; Radwan 2005; de la Fuente 2015; Medina 2015) in den letzten drei Jahrzehnten aus verschiedenen Blickwinkeln sowohl theoretisch als auch empirisch mit der positiven Rolle der Bewusstheit im Lernprozess beschäftigt. Als Ausgangspunkt für die empirischen Untersuchungen diente Schmidts Noticing-Hypothese, wobei besonders die verschiedenen Bewusstheitsebenen (d. h. die Ebene des Noticing und die Ebene des Understanding) genauer theoretisch und empirisch getestet und untersucht wurden. Schmidt (1990) definiert die Bewusstheitsebene des Noticing als ,the conscious registration of the occurence of some event" (Schmidt 1990: 29). Bei der höheren Bewusstheitsebene, der Understanding-Ebene, werden dann zielsprachliche Elemente, die bereits bemerkt wurden, weiter metalinguistisch analysiert. In diesem Prozess wird versucht, Regeln und Generalisierungen über das Input zu entdecken, und diesbezügliche Hypothesen zu testen. Sowohl das Vorwissen (vgl. z. B. Calderón 2013) als auch die Motivation der Lernenden können ebenfalls eine Rolle spielen und Einfluss auf diesen Prozess haben, da es bei der höheren Bewusstheitsebene um eine bewusste Analyse des (sprachlich) Wahrgenommenen geht. Die Bewusstheitsebenen scheinen daher von besonderer Wichtigkeit für die 
Ivana Šarić Šokčević:

Die Beziehung zwischen der Bewusstheit und den Lehrmethoden beim Erlernen der (nicht) passivfähigen Verben im Deutschen als Fremdsprache

Erforschung der Rolle der Bewusstheit im fremdsprachlichen Lernprozess zu sein, da sie die Aufteilung der Bewusstheit in die zugrunde liegenden Teilkomponenten ermöglichen.

\subsection{Forschungsinstrumente zur Untersuchung der Bewusstheitsebenen}

Um die interne Validität ${ }^{2}$ einer Untersuchung zu gewährleisten, und somit die Glaubwürdigkeit der Untersuchungsresultate zu maximieren, stellen in der aktuellen Erforschung der Rolle der Bewusstheit im Lernprozess nicht nur verschiedene theoretische Ansätze eine Herausforderung dar, sondern auch empirische und die damit verbundenen methodologischen Schwierigkeiten. Leow (2012: 3) betont in seinen Auslegungen diesbezüglich, dass man bei der Operationalisierung der Bewusstheit folgende methodologische Überlegungen anstellen müsse: ,(a) where awareness is measured (at the stage of encoding that is online versus at the stage of retrieval that is offline), (b) what kind of item is being targeted, and (c) how awareness is measured (the measurement instrument) “. Die Methode des Lauten Denkens oder die Think-aloud-Protokolle (engl. think aloud protocol) haben sich diesbezüglich als besonders nützlich erwiesen. Diese Methode dient zur Erhebung der kognitiven Prozesse und zur Verbalisierung der Gedanken, wobei man zwei verschiedene Arten dieser Think-aloud-Protokolle unterscheidet: (1) online Thinkaloud-Protokolle, bei denen der verbalisierte Gedankenstrom der Lernenden aufgenommen wird, während sie eine Aufgabe laut zu lösen versuchen, und (2) offline Think-aloud-Protokolle, die nach der Anwendung einer bestimmten Lernbedingung eingesetzt werden. Leow, Johnson und Zárate-Sández (2011) heben bezüglich des unterschiedlichen Einsatzes der online und offline Think-aloud-Protokolle im Bereich der Bewusstheitserforschung im Zweit- und Fremdsprachenunterricht folgendes hervor:

Where awareness is investigated is determined by whether measurement is performed concurrently (at the stage of construction) via online data-elicitation procedures or nonconcurrently (at the stage of reconstruction) via offline data elicitation

\footnotetext{
${ }^{2}$ Leow (2015:113) definiert interne und externe Validität wie folgt: "Two crucial features of a robust research design are the levels of the internal validity and external validity of the study itself. Internal validity deals with whether the interpretation of the research findings is firmly based within the study itself or, in other words, how well the data elicited answer the research questions (Hatch \& Lazaraton 1991). External validity deals with whether the findings can be generalizable to the participant population and other similar settings. A study cannot have external validity if it does not have internal validity." (Leow 2015: 110)
} 
procedures. As reported (...), the non-SLA fields has predominantly chosen/targeted the reconstruction stage for their measurement while the SLA field has opted, for the most part, to measure awareness at the stage of construction, that is, concurrently. (Leow, Johnson und Zárate-Sández 2011: 70)

Leow, Johnson und Zárate-Sández betonen hier nochmals, dass es von großer Wichtigkeit ist, sich bewusst zu machen und dann auch klar methodologisch zu artikulieren, warum und in welcher Lernphase Think-aloud-Protokolle eingesetzt werden, und welches Ziel vom Untersuchungsleiter damit verfolgt wird. Bei der Erforschung der Bewusstheitsebenen im Labor werden besonders die online Thinkaloud-Protokolle bevorzugt, da diese zur präziseren Erhebung der kognitiven Mikroprozesse, die sich im Lernenden während der Verarbeitung des neuen zielsprachlichen Lernmaterials abspielen, führen. Nach der Vollziehung dieser kognitiven Mikroprozesse, entstehen entsprechende Endprodukte im Lernprozess (siehe Abbildung 1), die ebenfalls gemessen und dann zur weiteren Verarbeitung bereitgestellt werden können. Leow (2015) plädiert jedoch an dieser Stelle besonders für die Ausdifferenzierung dieser beiden doch sehr unterschiedlichen Messinstrumente, um die daraus gewonnenen Resultate besser analysieren zu können und somit die Rolle der Bewusstheitsebenen in den verschiedenen Phasen des fremdsprachlichen Lernprozesses besser erfassen zu können. Zieht man jedoch in Betracht, dass Zweit- und Fremdsprachenforscher generell nicht nur an theoretischen Auslegungen und empirischen Untersuchungsergebnissen aus dem Labor für die Ausarbeitung der pädagogischen Implikationen interessiert sind, sondern auch an Untersuchungsergebnisse im schulischen Kontext, im Klassenzimmer, scheinen sowohl online als auch offline Think-aloud-Protokolle nützlich zu sein, um die im Labor gewonnen Ergebnisse zu testen.

\subsection{Empirische Untersuchungen bezüglich der Bewusstheit und der ex- pliziten und impliziten Lehrmethoden}

Die Variable Bewusstheit war Gegenstand sowohl psychologischer als auch sprachwissenschaftlicher Untersuchungen, wobei man sich diesbezüglich besonders damit beschäftigte, wie das Verstehen der fremdsprachlichen Zielstruktur am effektivsten angekurbelt werden kann und somit die Bewusstheit und die damit verbundenen Bewusstheitsebenen gefordert und gefördert werden können. Im Mittelpunkt dieser Untersuchungen standen daher auch das implizite und explizite Lernen und die impliziten und expliziten Lehrmethoden (DeKeyser 2003; Ellis, R. 2005) als zwei verschiedene Möglichkeiten, wie das Verstehen eines zielsprachlichen Inputs vollzogen werden kann. Das Verstehen eines fremdsprachlichen Inputs 
Ivana Šarić Šokčević:

Die Beziehung zwischen der Bewusstheit und den Lehrmethoden beim Erlernen der (nicht) passivfähigen Verben im Deutschen als Fremdsprache

kann durch explizites (extrinsisch motiviertes, nicht automatisiertes, faktenorientiertes) oder implizites (intrinsisch motiviertes, unbewusstes, fertigkeitsorientiertes, automatisiertes) Lernen erreicht werden (Schmidt 1990, 1994; Robinson 1996; Bagarić 2001; DeKeyser 2003; Ellis, R. 2005; Leow 2015). Unter explizitem und implizitem Wissen versteht man verschiedene Typen von linguistischem Wissen. Das Wissen über Sprache, oder explizites Sprachwissen, entsteht als Folge eines bewussten, kontrollierten und aufmerksamen Zugriffs auf sprachliche Phänomene (sprachliches Input) und ist mit der höheren Bewusstheitsebenen und metalinguistischem Wissen in Verbindung zu bringen, da die Absicht des Lernenden besteht, etwas zu erlernen (vgl. Bagarić 2001). Implizites (Sprach-)Wissen hingegen wird als intuitives Wissen definiert, das automatisiert ist, und, wenn überhaupt, auf einer niedrigen Bewusstheitsebene (vgl. Ellis, R. 2015: 7) basiert. Die Dichotomien explizites/implizites Wissen und explizites/implizites Lernen gehen auf die kognitive Linguistik und die im Bereich der Psychologie durchgeführten Experimente von Reber $(1967 ; 1969)$ bezüglich des impliziten Lernens Ende der 60-er Jahre. Diese theoretischen Ansätze bezüglich des impliziten und expliziten Wissens wurden durch empirische Studien (z. B. Anderson 1982; Green und Hecht 1992; Alderson und A. 1997; DeKeyser 1997; Han und Ellis 1998; Renou 2001; White und Ranta 2002; Elder und Manwaring 2004; Roehr 2007) erforscht. Im Mittelpunkt dieser Debatte steht die Beziehung zwischen explizitem und implizitem Lernen/Wissen und der Bewusstheit. Schmidt $(1990 ; 1995 ; 2001)$ aber auch andere Fremdsprachentheoretiker und -wissenschaftler (z. B. Leow 1997, 2015; Rosa und Leow 2004) betonen, dass explizites/implizites Lernen einen Prozess, wohingegen explizites/implizites Wissen ein Produkt darstellt (vgl. Ellis, R. 2015: 6).

Die Begriffe explizite und implizite Lehrmethoden gehen auf Michael Long zurück, der Anfang der 1990-er Jahre den Fokus-auf-Form-Ansatz definierte. Dieser Ansatz beschäftigte sich im fremdsprachlichen Sinne mit der Verarbeitung des fremdsprachlichen Inputs und versuchte sowohl formalsprachliche als auch inhaltsbezogene Aspekte zu vereinigen (vgl. auch Doughty und Williams 1998: 2). Bei diesem Konzept stehen die bewusste und kontrollierte Sprachverarbeitung und Steuerung sprachlichen Inputs im Mittelpunkt. Das Resultat verschiedener Diskussionen bezüglich der fremdsprachendidaktischen Realisierungsmöglichkeiten im Rahmen eines solchen Fokus-auf-Form-Ansatzes waren empirische Untersuchungen (vgl. Doughty und Williams 1998; Lightbown 1998, 2000; Ellis R. 2000; Norris und Ortega 2000), die die Wirksamkeit verschiedener Ansätze und die dazugehörigen Instruktionsverfahren erforschten. Eine der wichtigsten Unterteilungen bezüglich der Instruktionsmaßnahmen im Rahmen des Fokus-auf-Form-Ansatzes ist das explizit-implizite Kontinuum, bzw. die expliziten (z. B. Verarbeitungsanlei- 
tung, sprachbewusstheitsfördernde Aufgaben usw.) und impliziten (z. B. Inputflut, Inputintensivierung usw.) Instruktionen.

Die Aufteilung des fremdsprachlichen Lernprozesses in kleinere Mikroprozesse und die Aufteilung der expliziten und impliziten Instruktionen in die entsprechenden Mikromaßnahmen und -verfahren war Anlass zu zahlreichen empirischen Untersuchungen. Diese empirischen Untersuchungen wurden hauptsächlich im Labor durchgeführt, doch es gibt auch Untersuchungen, die das Augenmerk auf den natürlichen pädagogischen Kontext, das Klassenzimmer, gesetzt haben (z. B. Radwan 2005; Ammar 2008). Man ist intensiv der Frage nachgegangen, welche der Instruktionsmaßnahmen, die sich auf dem explizit-impliziten Instruktionskontinuum befinden, die Bewusstheitsebenen ankurbeln und somit zu einem effektiveren Lernprozess beitragen können. Die durchgeführten empirischen Untersuchungen weisen verschiedene Ziele und Ansätze auf, weshalb diese nach verschiedenen Kriterien aufgeteilt werden können: So kann z. B. zwischen Laboruntersuchungen (z. B. Scott 1989, 1990; Doughty 1991; DeKeyser 1995; Alanen 1995; Hulstijn und A. 1996; Robinson 1995, 1996, 1997; de Graaff 1997; Williams 1999) und Klassenzimmerstudien (z. B. Doughty 1991; White 1991; DeKeyser 1993; Fotos 1993; Alanen 1995; Ellis, R. 1995; Winitz 1996; Radwan 2005; Ammar 2008) unterschieden werden. Diese Untersuchungen weisen auch Unterschiede hinsichtlich der Zielsprache auf, da der Untersuchungsgegenstand dieser Studien sowohl natürliche (besonders fremdsprachliche Zielstrukturen aus dem Englischen, Spanischen und Finnischen) als auch künstliche Sprachen waren (vgl. Leow 2015). Es wurden außerdem auch verschiedene morphologische, syntaktische, aber auch semantische und lexikalische Zielstrukturen untersucht. Das Ziel dieser Untersuchungen war es, die Interaktion zwischen den expliziten und impliziten Instruktionsmaßnahmen und der Bewusstheit zu erforschen. Es wurde die Rolle der Bewusstheit und der Bewusstheitsebenen der Lernenden bezüglich bestimmter fremdsprachlicher Zielstrukturen erforscht, während die Lernenden expliziten und impliziten Instruktionsmaßnahmen ausgesetzt waren. Diese Untersuchungen haben auch einige spezifische Merkmale der Lernenden thematisiert, wie beispielsweise den Wissensstand (engl. level of proficiency) der Lernenden (niedrigerer Wissensstand vs. höherer Wissensstand) (z. B. Gass, Svetics, Lemelin 2003; Calderón 2013) oder auch die Komplexität der untersuchten fremdsprachlichen Zielstruktur (einfache vs. komplexe Zielstrukturen) (wie z. B. DeKeyser 1995; Williams 1999; Andrews 2007). Diese Untersuchungsergebnisse lassen schlussfolgern, dass die Bewusstheit eine wichtige Rolle in den frühen Phasen der Inputverarbeitung zu spielen scheint, wobei die Bewusstheitsebenen eine starke positive Korrelation mit besseren Lernergebnissen, besonders derjenigen Lernenden, die expliziten Lehr- 
methoden ausgesetzt waren, und der niedrigeren als auch höheren Bewusstheitsebene aufweisen (Radwan 2005; Andrews 2007). Schließlich muss auch festgehalten werden, dass eine der größten Schwierigkeiten beim Vergleich dieser Untersuchungen noch immer die Tatsache ist, dass es zwar viele vereinzelte empirische Untersuchungen gibt, die sich mit den Variablen Bewusstheitsebenen und explizite/implizite Instruktionsmaßnahmen beschäftigt haben, jedoch immer noch sehr wenige Studien, die die Ergebnisse dieser empirischen Untersuchungen aus Laborstudien im Klassenzimmer getestet haben (DeKeyser 2003; Radwan 2005). Da das Ziel jeglicher Fremd- und Zweitsprachenerforschung langfristig das Implementieren neuer pädagogischer Implikationen sein sollte, gibt es Bedarf an genau solchen Untersuchungen (Radwan 2005; Andrews 2007). Bezüglich der Laboruntersuchungen, des expliziten/impliziten Lernens und der Bewusstheit gibt es schon einige Replikationsuntersuchungen (z. B. Leow 1998; Rosa und Leow 2004), bei denen die empirisch gewonnen Daten nochmals hinterfragt und getestet wurden, dies ist jedoch für die Untersuchungen im Klassenzimmer wegen des methodologischen Aufwands noch immer nicht der Fall. Die vorliegende Untersuchung versucht diese Lücken zu füllen.

\section{Methodologie}

\subsection{Untersuchungsgegenstand}

Die Zielstruktur der vorliegenden Untersuchung sind (nicht) passivfähige Verben im deutschen Vorgangspassiv. Diese Zielstruktur wurde aus mehreren Gründen ausgewählt. Der erste Grund bezieht sich auf die Komplexität dieser Struktur. Es geht um eine komplexe, mehrdimensionale Struktur, die sowohl die morphologische, syntaktische als auch semantisch-pragmatische Ebene umfasst. Der zweite Grund ist die Tatsache, dass es keinerlei uns zugängliche Untersuchungen bezüglich der Bewusstheit im Prozess des Erlernens der (nicht) passivfähigen Verben gibt. Außerdem bietet sich die Zielstruktur zur Erforschung der für diese Untersuchung ausgewählten Zielgruppen aus zwei Perspektiven an: Die erste Untersuchungsgruppe, die Gymnasialschüler, befinden sich auf einem niedrigeren Wissensstand, und bei der zweiten Untersuchungsgruppe, den Germanistikstudenten, kann man von einer höheren Motivation und einem besseren Vorwissen ausgehen. Für beide Teilnehmergruppen ist die ausgewählte Zielstruktur unbekannt, was Grundlage zur Erforschung der Bewusstheitsebenen in der Input-intake-Phase bietet. Außerdem werden beide Gruppen trotz ihrer verschiedenen Merkmale in der Lage sein, diese für sie unbekannte Zielstruktur auf ihrem gegenwärtigen Spra- 


\section{EZIKOSLOVLJE \\ 22.1 (2021): 99-148}

chentwicklungsniveau zu verarbeiten, was eine zusätzliche Möglichkeit des Vergleichs bietet.

\subsection{Untersuchungsziel, -fragen und-hypothesen}

Das Hauptziel der Untersuchung ist es, die Beziehung zwischen den Bewusstheitsebenen und den expliziten und impliziten Lehrmethoden in der frühen Phase des Lernprozesses, der Intake-input-Phase, der (nicht) passivfähigen Verben im Deutschen zu untersuchen. Es wird somit versucht, die folgenden komplexen Fragen zu beantworten und den folgenden Hypothesen nachzugehen:

Untersuchungsfrage 1: In welcher Beziehung stehen die Lernergebnisse des unmittelbaren und verzögerten Posttests und die Bewusstheitsebenen bei denjenigen Untersuchungsteilnehmern, die expliziten Lehrmethoden ausgesetzt waren, und denjenigen, die impliziten Lehrmethoden ausgesetzt waren?

Untersuchungsfrage 2: In welcher Beziehung stehen die Lernergebnisse des unmittelbaren und verzögerten Posttests und die Bewusstheitsebenen bei den Germanistikstudenten im Gegensatz zu den Gymnasiasten?

Hypothese 1: Die Lernergebnisse des unmittelbaren und verzögerten Posttests bei denjenigen Untersuchungsteilnehmern, die expliziten Lehrmethoden ausgesetzt waren, werden eine stärkere Interaktion bezüglich der beiden Bewusstheitsebenen aufweisen als diejenigen Untersuchungsteilnehmer, die impliziten Lehrmethoden ausgesetzt waren.

Hypothese 2: Die Lernergebnisse der Germanistikstudenten werden beim unmittelbaren und verzögerten Posttest unabhängig von der Art der Lehrmethode eine Interaktion sowohl mit der niedrigeren als auch mit der höheren Bewusstheitsebene aufweisen im Gegensatz zu den Lernergebnissen der Gymnasiasten.

\subsection{Untersuchungsdesign}

\subsubsection{Teilnehmer}

Die 92 Untersuchungsteilnehmer waren kroatische Gymnasiasten der dritten Klasse eines vierjährigen Gymnasialprogrammes und kroatische Germanistikstudenten des ersten Studienjahres eines Bachelorprogrammes. Die erste Untersuchungsgruppe, die Gymnasiasten, befinden sich laut dem Gemeinsamen europäischen Refe- 
renzrahmen $(\mathrm{GeR})^{3}$ auf der Sprachebene B1/B1+. Man geht in dieser Untersuchung davon aus, dass sie keinerlei formale Vorkenntnisse bezüglich der Zielstruktur besitzen, was jedoch auch noch vor Beginn der Untersuchung getestet werden wird. Laut der Angaben ihrer Deutschlehrerin lernen alle Untersuchungsteilnehmer zwei Stunden pro Woche Deutsch als Fremdsprache. Die Gruppe der Gymnasiasten $(\mathrm{N}=43)$ wurde nach der Klasse, die sie besuchen, in zwei Untergruppen unterteilt, eine explizite Untersuchungsgruppe und eine implizite Untersuchungsgruppe. Die beiden Klassen unterscheiden sich nach dem Notendurchschnitt im Fach Deutsch am Ende der zweiten Klasse kaum voneinander. ${ }^{4}$

In der zweiten Untersuchungsgruppe, der Gruppe der Germanistikstudenten, haben alle zuvor das kroatische Abitur im Fach Deutsch abgelegt, sie sollten sich daher alle laut dem GeR auf der Sprachebene B2/B2+ befinden. Es ist anzunehmen, dass sie in Bezug auf die Zielstruktur über ein gewisses implizites Vorwissen bezüglich der (Nicht-)Akzeptanz des sprachlichen Gebrauchs bestimmter Verben im Vorgangspassiv verfügen. Dieses Sprachbewusstsein ist durch das Interesse und die intensivere kommunikative Nutzung der deutschen Sprache auf einer höheren Sprachebene zu erklären. Die Untersuchungsgruppe der Studenten $(\mathrm{N}=49)$ wurde ebenfalls in zwei Untergruppen unterteilt, eine explizite und eine implizite. Zuvor wurden sie jedoch einem gängigen Einstufungstest unterzogen, um die gleichmäßige Aufteilung in Gruppen zu gewährleisten.

\subsubsection{Untersuchungsmethoden und -instrumente}

Zur Datenerhebung wurden in dieser Untersuchung die folgenden Methoden angewandt: das Experiment und die Think-aloud-Methode. Als Forschungsinstrument wurde ein Pretest, ein unmittelbarer Posttest und ein verzögerter Posttest verwendet.

Das Experiment bestand aus drei Phasen: einem Pretest, einer Treatmentphase $\mathrm{e}^{5}$ mit einem unmittelbaren (engl. immediate) Posttest und einem verzögerten (engl. delayed) Posttest. Das Ziel der ersten Phase, des Pretests ${ }^{6}$, war es bei den Unter-

\footnotetext{
${ }^{3} \mathrm{https}: / /$ www.coe.int/en/web/common-european-framework-reference-languages (abgerufen am 4. Juli 2021)

${ }^{4}$ Durchschnittsnote für die Klasse $1=3,78$ mit einer Standardabweichung von 0,72; Durchschnittsnote für Klasse $2=3,81$ mit einer Standardabweichung von 0,84 .

${ }^{5}$ Siehe Anhang 4

${ }^{6}$ Siehe Anhang 1
} 


\section{EZIKOSLOVLJE \\ 22.1 (2021): 99-148}

suchungsteilnehmern den Ausgangspunkt bezüglich der Bewusstheit über die Zielstruktur festzulegen.

In der zweiten Phase, der Treatmentphase mit einem unmittelbaren Posttest, wurden die beiden Untersuchungsgruppen in zwei Untergruppen unterteilt. Die erste Untergruppe wurde während der Treatmentphase expliziten Lehrmethoden ausgesetzt, wohingegen die zweite Untergruppe impliziten Lehrmethoden unterzogen wurde. Zur Auswahl der einzelnen impliziten und expliziten Instruktionen wurden für diese Untersuchung Arbeit die Metaanalysen von Norris und Ortega (2000), Spada und Tomita (2010) und Goo, Granena, Yilmaz und Novella (2015) herangezogen. Die in diesen Arbeiten untersuchten und verwendeten Instruktionsmaßnahmen wurden analysiert und nach dem Kriterium der Häufigkeit und dem Kriterium des Grades der Explizitheit/Implizitheit für die vorliegende Untersuchung Arbeit herausgesucht. Um die vorliegende Studie später mit anderen besser vergleichen zu können, wurden diejenigen Instruktionsmaßnahmen herausgesucht, die in diesen Metaanalysen am häufigsten vorkamen. Das Kriterium bezüglich des Grades der Explizitheit/Implizitheit bezieht sich auf diejenigen Instruktionsmaßnahmen, die sich auf dem expliziten-impliziten Kontinuum als entgegengesetzte Instruktionspole befinden. Unter der Berücksichtigung der Erkenntnisse der angeführten Metaanalysen und in Anlehnung an weitere relevante Untersuchungen aus den Zweit- und Fremdsprachensträngen, die sich mit der positiven Rolle der Bewusstheit im Lernprozess beschäftigen (z. B. Leow 1997; 2015; Rosa und O'Neill 1999; Andrews 2007), wurden für die vorliegende Arbeit die folgenden expliziten und impliziten Instruktionsmaßnahmen ausgewählt:

1. Explizite Instruktionsmaßnahmen: Formpräsentation oder grammatische Regelerklärung (wie bei z. B. Benati 2005; Master 1994), kontrastiver Ansatz (Mutter- vs. Fremdsprache) (wie bei z. B. Spada und A. 2005), explizites Feedback (metalinguistisches Feedback und explizite Fehlerkorrektur) (wie bei z. B. Ellis und A. 2006; Carroll und Swain 1993).

2. Implizite Instruktionsmaßnahmen: „Wenn das Treatment keine Regelpräsentation bezüglich der Zielstruktur oder Anleitung, nach einer Regel zu suchen, beinhaltet, spricht man von impliziten Instruktionen" (Norris und Ortega 2000: 437), Inputflut (wie bei z. B. Spada und Lightbown 1999; Williams und Evans 1998), Inputverstärkung, implizites Feedback (Reformulierung und Wiederholung).

Unmittelbar nach der Treatmentphase wurden die Bewusstheitsebenen der Untersuchungsteilnehmer mit einem unmittelbaren Posttest $^{7}$ erfragt, um die

\footnotetext{
${ }^{7}$ Siehe Anhang 2
} 
Bewusstheitsebenen nach der Treatmentphase zu erforschen und somit mögliche Veränderungen festhalten zu können.

In der dritten Phase, die bei beiden Untersuchungsgruppen nach mehr als zwei Monaten nach der Treatmentphase und dem unmittelbaren Posttest stattfand, wurde ein verzögerter Posttest ${ }^{8}$ durchgeführt. Aufgrund der Tatsache, dass die Beispiele bezüglich der fremdsprachlichen Zielstruktur, die für die Untersuchungsteilnehmer aufgrund ihrer Leistungsstufe und anderer, für diese Untersuchung wichtigen Kriterien angemessen sind, limitiert sind, entschied man sich dafür, den gleichen Test wie beim Pretest zu verwenden. Soweit uns bekannt ist, konnte kein Reaktivitätseffekt bezüglich der Beispiele mithilfe der Think-aloud-Protokolle festgehalten werden.

\subsubsection{Think-aloud-Methode}

Das Ziel der Datenerhebung mithilfe der Think-aloud-Protokolle ist es, zu untersuchen, auf welcher Bewusstheitsebene sich die Untersuchungsteilnehmer bezüglich der Zielstruktur in den einzelnen Phasen der Untersuchung befinden. Es muss jedoch an dieser Stelle betont und erläutert werden, dass die Think-aloudProtokolle nicht während der Treatmentphase (engl. online), sondern nach der Treatmentphase (engl. offline) und während der Testphase angewandt wurden. In der vorliegenden Untersuchung konnten aus mehreren Gründen nicht die online Thinkaloud-Protokolle angewandt werden, obwohl diese in den Laboruntersuchungen stark bevorzugt wurden (vgl. Leow 2015). Der Hauptgrund ist der Kontext, in dem die vorliegende Untersuchung durchgeführt wurde. Die Untersuchungsteilnehmer sind kroatische Gymnasiasten und Germanistikstudenten, die noch nie an einer sprachwissenschaftlichen Untersuchung teilgenommen haben. Darüber hinaus ist das kroatische Schulsystem in Bezug auf das Erlernen des Deutschen als Fremdsprache noch immer lehrerorientiert und wird in einem expliziteren Kontext erlernt. Es wird versucht durch das Produkt, das durch die Umwandlung von Input in Intake entsteht und die Verarbeitungsprozesse in eben dieser ersten Phase, die Rolle der Bewusstheit zugänglich zu machen, zu messen und somit weiter ihre Rolle zu erforschen. Es wird daher das Intake als Produkt in der zweiten Phase des Lernprozesses gemessen. Die vorliegende Untersuchung hätte auch in einem Labor durchgeführt werden können, was die Erforschung des Prozesses und nicht des Produktes ermöglicht hätte. Es besteht jedoch Bedarf an empirischen Untersuchungen, welche die bereits erforschte Rolle und die entsprechenden empirischen Ergebnisse

\footnotetext{
${ }^{8}$ Siehe Anhang 3
} 
bezüglich der Bewusstheit in Laboruntersuchungen in einem natürlichen Kontext testen, ,unless we do so, the results will remain tenuous, speculative, and often times contentious“ (Radwan 2005: 84). Die Think-aloud-Protokolle des Pretests, des unmittelbaren und verzögerten Posttests wurden am Ende der Untersuchung von der Untersuchungsleiterin transkribiert.

\subsubsection{Datenerhebung}

In der ersten Phase wurde der Pretest durchgeführt, der auf einem Computer mithilfe eines Googleformulars für die Untersuchungsteilnehmer bereitgestellt wurde. Der Pretest wurde jeweils eine Woche vor dem Treatment in den Computerräumen der jeweiligen Institution ausgefüllt. Der Pretest dauerte insgesamt ca. 45 Minuten, wobei die Untersuchungsteilnehmer zeitlich auf 30 Minuten für die Lösung des Pretests beschränkt waren. Die Think-aloud-Protokolle wurden mit Hilfe des Programmes Audacity aufgenommen. Die Aufgabe der Untersuchungsteilnehmer war es, ihre Gedanken bezüglich der grammatischen Korrektheit und sprachlichen Akzeptanz der Zielstrukturen laut ins Mikrophon zu verbalisieren. Auf diese Weise wurde versucht, das Verarbeiten der präsentierten Zielstrukturen und somit der möglichen Bewusstheitsebenen für die Untersuchungsleiterin zugänglich zu machen.

In der zweiten Phase wurde das Treatment durchgeführt, das in beiden Untersuchungsgruppen und den jeweiligen Untergruppen jeweils 90 Minuten dauerte. Die Treatmentphase wurde für alle Gruppen von derselben Untersuchungsleiterin durchgeführt, um die interne Validität der Untersuchung zu gewährleisten. Nach dem Treatment wurde sofort der unmittelbare Posttest durchgeführt, der ebenfalls 30 Minuten dauerte.

In der dritten Phase, die nach mehr als zwei Monaten nach dem Treatment stattfand, wurde der verzögerte Posttest durchgeführt, der ebenfalls 30 Minuten dauerte.

\subsubsection{Methoden der qualitativen und quantitativen Datenanalyse}

Die erhobenen Daten wurden qualitativ und quantitativ analysiert. Zuerst wurden die Lernergebnisse der einzelnen Tests ausgewertet. Danach wurden die aufgenommenen Think-aloud-Protokolle transkribiert und hinsichtlich der Bewusstheitsebenen analysiert und kodifiziert. Die beiden Bewusstheitsebenen wurden mit $[+\mathrm{N}]$ für Noticing (die niedrigere Bewusstheitsebene) oder [+U] für Understanding 
Ivana Šarić Šokčević:

Die Beziehung zwischen der Bewusstheit und den Lehrmethoden beim Erlernen der (nicht) passivfähigen Verben im Deutschen als Fremdsprache

(die höhere Bewusstheitsebene) kodifiziert. Als Kriterium für die Kodifizierungen wurden die von Leow (2015: 228) festgelegten Deskriptoren für das verbalisierte Verhalten der Lernenden herangezogen:

1. Die niedrigere Bewusstheitsebene, die Noticing-Ebene [+N]: Der Lernende liest die Zielstruktur langsam, betont sie aufmerksam, wiederholt sie, übersetzt diese in die Muttersprache, ist sich darüber nicht im Klaren, wie die Zielstruktur zu verstehen ist, beschäftigt sich nicht zu lange mit der Zielstruktur.

2. Die höhere Bewusstheitsebene, die Understanding-Ebene $[+\mathrm{U}]$, wird mit der metalinguistischen Bewusstheit in Verbindung gebracht. Dabei stellt der Lernende Hypothesen bezüglich des Lerngegenstandes auf, beschäftigt sich länger mit der Zielstruktur, korrigiert eigene Übersetzungen, die er zuvor angefertigt hatte, zeigt eine hohe Ebene an kognitiver Anstrengung mit dem Ziel der grammatischen Verarbeitung der Zielstrukturen, diese Anstrengungen resultieren in der Formulierung einer vollständig korrekten Regel.

Nach der qualitativen Analyse aller Daten wurde die statistische Analyse der von der Untersuchung umfassten Variablen vorgenommen: Test (Pretest vs. unmittelbarer Posttest vs. verzögerter Posttest), Lehrmethode (explizit vs. implizit), Bewusstheitsebene (niedrigere vs. höhere) und Leistungsstufe (Fakultät vs. Gymnasium). Um diese Variablen statistisch zu untersuchen, wurde eine mehrfaktorielle Varianzanalyse (ANOVA mit Greenhouse-Geisser-Korrektur und ANOVA posthoc Tests mit Bonferroni Korrektur) durchgeführt.

\section{Untersuchungsergebnisse}

\subsection{Ergebnisse der statistischen Analyse}

In Bezug auf die erste Untersuchungsfrage wurde zuerst statistisch der Beziehung zwischen den Lernergebnissen derjenigen Untersuchungsteilnehmer, die expliziten Lehrmethoden ausgesetzt waren, und den Lernergebnissen derjenigen, die impliziten Lehrmethoden unterzogen wurden, beim Pretest und unmittelbaren Posttest nachgegangen.

Tabelle 1 zeigt die F-Werte einer mixed ANOVA-Analyse mit Messwiederholung mit Greenhouse-Geisser-Korrektur für die Variable Test (Pretest vs. unmittelbarer Posttest) und den Interaktionseffekt zwischen den Variablen Test (Pretest vs. unmittelbarer Posttest) und Lehrmethode (explizit vs. implizit). 


\section{EZZIKOSLOVLE \\ 22.1 (2021): 99-148}

Tabelle 1. F-Werte der mixed ANOVA mit Messwiederholung mit Greenhouse-GeisserKorrektur für die Variable Test (Pretest vs. unmittelbarer Posttest) und in Bezug auf die Variablen Lehrmethode (explizit vs. implizit) und Leistungsstufe (Fakultät vs. Gymnasium)

\begin{tabular}{|l|c|c|r|}
\hline Variable & F $(1,86)$ & Sig. & Partielles Eta-Quadrat $^{9}$ \\
\hline Test & 312,912 &, 000 &, 784 \\
\hline Test*Lehrmethode & 125,234 &, 000 &, 593 \\
\hline
\end{tabular}

Die abhängige Variable Test $(\mathrm{F}(1,86)=312,91), \mathrm{p}<, 01$, partielles $\left.\varepsilon^{2}=, 78\right)$ zeigt einen signifikanten Haupteffekt mit einer sehr starken Effektgröße. Zudem zeigt sich ein statistisch signifikanter Interaktionseffekt zwischen der Variable Lehrmethode $(\mathrm{F}(1,86)=125,23), \mathrm{p}<, 01$, partielles $\left.\varepsilon^{2}=, 59\right)$ und der Variable Test. Für die Variablen, die eine signifikante Interaktion aufzeigen, wurde ein Bonferronikorrigierter post-hoc Test (siehe Tabelle 2, 3) durchgeführt, um die Unterschiede und Interaktionen genauer zu untersuchen. Der Bonferroni-korrigierte post-hoc Test für die Variable Test (Pretest vs. unmittelbarer Posttest) (siehe Tabelle 2) zeigt einen signifikanten Unterschied in der Performanz zwischen den Resultaten des Pretests und des unmittelbaren Posttests, d. h. dass die Resultate der Untersuchungsteilnehmer statistisch besser beim unmittelbaren Posttest (-6,89, 95\% - CI [-7,67, 6,12]) als beim Pretest waren.

Tabelle 2. Bonferroni-korrigierter post-hoc Test für die Variable Test (Pretest vs. unmittelbarer Posttest)

\begin{tabular}{|c|c|c|c|c|c|c|}
\hline \multirow{2}{*}{\multicolumn{2}{|c|}{ Test }} & \multirow[t]{2}{*}{$\begin{array}{l}\text { Mittlere Diffe- } \\
\text { renz }\end{array}$} & \multirow[t]{2}{*}{$\begin{array}{l}\text { Standard- } \\
\text { fehler }\end{array}$} & \multirow[t]{2}{*}{ Sig. ${ }^{b}$} & \multicolumn{2}{|c|}{$\begin{array}{l}\text { 95\% Konfidenzintervall } \\
\text { für } \\
\text { die Differenz } \\
\end{array}$} \\
\hline & & & & & Untergr. & Obergr. \\
\hline Pretes & Unmittelbarer Posttest & $-6,891^{*}$ &, 390 &, 000 & $-7,666$ & $-6,117$ \\
\hline
\end{tabular}

Basiert auf den geschätzten Randmitteln; *. Die mittlere Differenz ist auf dem ,05-Niveau signifikant; b. Anpassung für die Mehrfachvergleiche: Bonferroni

Die in Tabelle 1 angedeutete signifikante Interaktion zwischen den Variablen Test (Pretest vs. unmittelbarer Posttest) und Lehrmethode (explizit vs. implizit) $(\mathrm{F}(1,86)$ $=125,23), \mathrm{p}<, 01$, partielles $\left.\varepsilon^{2}=, 59\right)$ hat beim Bonferroni-korrigierten post-hoc Test (siehe Tabelle 3) eine signifikante Interaktion zwischen dem unmittelbaren Posttest und den Ergebnissen der Untersuchungsteilnehmer, die expliziten Lehrmethoden ausgesetzt waren, aufgezeigt (8,55, 95\% - CI [7,09, 10,02]).

\footnotetext{
${ }^{9}$ In dieser Arbeit wird die Effektstärke mit denen nach Cohen (1988) festgelegten Werten interpretiert: partielles $\varepsilon^{2:}$ von $0.100=$ kleiner Effekt; von 0.243 = mittlerer Effekt; von 0.372 = starker Effekt.
} 


\section{Ivana Šarić Šokčević: \\ Die Beziehung zwischen der Bewusstheit und den Lehrmethoden beim Er- lernen der (nicht) passivfähigen Verben im Deutschen als Fremdsprache}

Ferner wurde statistisch der Beziehung zwischen den Lernergebnissen, den Bewusstheitsebenen und der Lehrmethode beim unmittelbaren und verzögerten Posttest auf den Grund gegangen. Es wurde diesbezüglich eine statistische Analyse bezüglich der Variablen Bewusstheitsebene (niedrigere vs. höhere), Lehrmethode (explizit vs. implizit) und Test (unmittelbarer vs. verzögerter Posttest) durchgeführt.

Tabelle 3. Bonferroni-korrigierter post-hoc Test für die Variablen Test (Pretest vs. unmittelbarer Posttest) und Lehrmethode (explizit vs. implizit)

\begin{tabular}{|c|c|c|c|c|c|c|c|}
\hline \multirow[t]{2}{*}{ Test } & \multirow[t]{2}{*}{$\begin{array}{c}\text { (I) } \\
\text { Lehrmeth. }\end{array}$} & \multirow[t]{2}{*}{$\begin{array}{c}(\mathrm{J}) \\
\text { Lehmeth. }\end{array}$} & \multirow[t]{2}{*}{$\begin{array}{l}\text { Mittlere Diffe- } \\
\text { renz } \\
\text { (I-J) }\end{array}$} & \multirow[t]{2}{*}{$\begin{array}{l}\text { Standard- } \\
\text { fehler }\end{array}$} & \multirow[t]{2}{*}{ Sig. ${ }^{b}$} & \multicolumn{2}{|c|}{$\begin{array}{c}\text { 95\% Konfidenzintervall } \\
\text { für } \\
\text { die Differenz }{ }^{\mathrm{b}} \\
\end{array}$} \\
\hline & & & & & & Untergr. & Obergr. \\
\hline Pretest & explizit & implizit &,- 165 & ,587 & 779 & $-1,331$ & 1,001 \\
\hline $\begin{array}{l}\text { Unmittelbarer } \\
\text { Posttest }\end{array}$ & explizit & implizit & $8,554^{*}$ & ,736 & , 000 & 7,091 & 10,018 \\
\hline
\end{tabular}

Basiert auf den geschätzten Randmitteln; *. Die mittlere Differenz ist auf dem ,05-Niveau signifikant; b. Anpassung für die Mehrfachvergleiche: Bonferroni

Die mixed ANOVA-Analyse (Tabelle 4) zeigt, dass die Variablen Bewusstheitsebene (niedrigere vs. höhere), Lehrmethode (explizit vs. implizit) und Test (unmittelbarer Posttest vs. verspäteter Posttest) mit unterschiedlichen Werten statistisch signifikant sind und in Interaktion stehen. Ein Bonferroni-korrigierter post-hoc Test wurde für diese Variablen durchgeführt, um den statistischen Unterschied zwischen den einzelnen Variablen und die Interaktionen weiter zu untersuchen.

Tabelle 4. F-Werte der mixed ANOVA mit Messwiederholung mit Greenhouse-GeisserKorrektur für die Variablen Bewusstheitsebene (niedrigere vs. höhere), Lehrmethode (explizit vs. implizit) und Test (unmittelbarer Posttest vs. verzögerter Posttest)

\begin{tabular}{|l|r|r|}
\hline Variable & F $(1,87)$ & Partielles Eta-Quadrat \\
\hline Test & $93,251^{* *}$ &, 517 \\
\hline Bewusstheitsebene & $8,884^{* *}$ &, 093 \\
\hline Lehrmethode & $115,715^{* *}$ &, 571 \\
\hline Bewusstheitsebene*Lehrmethode & $131,370^{* *}$ &, 602 \\
\hline Test*Bewusstheitsebene & $28,225^{* *}$ &, 245 \\
\hline Test*Bewusstheitsebene*Lehrmethode & $74,303^{* *}$ &, 461 \\
\hline
\end{tabular}

Der Bonferroni-korrigierte post-hoc Test (Tabelle 5) zeigt einen signifikanten Unterschied $(\mathrm{p}<, 01)$ in der Performanz zwischen den Ergebnissen derjenigen Untersuchungsteilnehmer, die expliziten Lehrmethoden ausgesetzt waren, und denjenigen, die impliziten Lehrmethoden unterzogen wurde, bezüglich der höheren 


\section{EZZIKOSLOVLE \\ 22.1 (2021): 99-148}

Bewusstheitsebene. Diejenigen Untersuchungsteilnehmer, die der expliziten Lehrmethode ausgesetzt waren, zeigen im Gegensatz zu den Untersuchungsteilnehmern, die der impliziten Lehrmethode unterzogen wurden, ein besseres Ergebnis $(6,02$, $95 \%$ - CI $[0,21,0,82])$ bezüglich der höheren Bewusstheitsebene.

Tabelle 5. Bonferroni-korrigierter post-hoc Test für die Variablen Bewusstheitsebene (niedrigere vs. höhere) und Lehrmethode (explizit vs. implizit)

\begin{tabular}{|c|c|c|c|c|c|c|c|}
\hline \multirow[t]{2}{*}{$\begin{array}{l}\text { Bewusstheits- } \\
\text { ebene }\end{array}$} & \multirow[t]{2}{*}{$\begin{array}{c}\text { (I) } \\
\text { Lehrmeth. }\end{array}$} & \multirow[t]{2}{*}{$\begin{array}{c}(\mathrm{J}) \\
\text { Lehrmeth. }\end{array}$} & \multirow{2}{*}{$\begin{array}{c}\text { Mittlere } \\
\text { Differenz } \\
\text { (I-J) }\end{array}$} & \multirow[t]{2}{*}{$\begin{array}{l}\text { Standard- } \\
\text { fehler }\end{array}$} & \multirow[t]{2}{*}{ Sig. ${ }^{b}$} & \multicolumn{2}{|c|}{$\begin{array}{l}\text { 95\% Konfidenzintervall für } \\
\text { die Differenz }^{\text {b }}\end{array}$} \\
\hline & & & & & & Untergr. & Obergr. \\
\hline niedrigere & explizit & implizit &,- 589 & ,427 & , 171 & $-1,438$ &, 259 \\
\hline höhere & explizit & implizit & $6,024^{*}$ & ,335 & ,000 & 5,359 & 6,689 \\
\hline
\end{tabular}

Basiert auf den geschätzten Randmitteln; *. Die mittlere Differenz ist auf dem ,05-Niveau signifikant;

b. Anpassung für die Mehrfachvergleiche: Bonferroni

Tabelle 6 zeigt auch eine statistische Relevanz $(\mathrm{p}<, 01)$ bezüglich der Interaktion zwischen der höheren Bewusstheitsebene und dem unmittelbaren Posttest $(2,85$, $95 \%$ - CI $[2,33,3,38])$.

Tabelle 6. Bonferroni-korrigierter post-hoc Test für die Variablen Bewusstheitsebene (niedrigere vs. höhere) und Test (unmittelbarer Posttest vs. verzögerter Posttest)

\begin{tabular}{|c|c|c|c|c|c|c|c|}
\hline \multirow[t]{2}{*}{$\begin{array}{l}\text { Bewusstheits- } \\
\text { ebene }\end{array}$} & \multirow[t]{2}{*}{$\begin{array}{l}\text { (I) } \\
\text { Test }\end{array}$} & \multirow[t]{2}{*}{$\begin{array}{l}\text { (J) } \\
\text { Test }\end{array}$} & \multirow[t]{2}{*}{$\begin{array}{l}\text { Mittlere } \\
\text { Differenz } \\
\text { (I-J) }\end{array}$} & \multirow[t]{2}{*}{$\begin{array}{l}\text { Standard- } \\
\text { fehler }\end{array}$} & \multirow[t]{2}{*}{ Sig. $^{b}$} & \multicolumn{2}{|c|}{$\begin{array}{c}95 \% \text { Konfidenzintervall } \\
\text { für } \\
\text { die Differenz }{ }^{\mathrm{b}}\end{array}$} \\
\hline & & & & & & Untergr. & Obergr. \\
\hline niedrigere & unmittelbar & verzögert & ,527 & 296 & 078 &,- 061 & 1,114 \\
\hline höhere & unmittelbar & verzögert & $2,851^{*}$ & 264 &, 000 & 2,327 & 3,375 \\
\hline
\end{tabular}

Basiert auf den geschätzten Randmitteln; *. Die mittlere Differenz ist auf dem ,05-Niveau signifikant; b. Anpassung für die Mehrfachvergleiche: Bonferroni.

Ein Bonferroni-korrigierter post-hoc Test (Tabelle 7) zeigt einen signifikanten Unterschied $(\mathrm{p}<, 01)$ zwischen der höheren und niedrigeren Bewusstheitsebene beim unmittelbaren Posttest bei denjenigen Untersuchungsteilnehmern, die den expliziten Lehrmethoden ausgesetzt waren, aber auch denjenigen, die den impliziten Lehrmethoden ausgesetzt waren. Beim unmittelbaren Posttest zeigen diejenigen Untersuchungsteilnehmer, die den impliziten Lehrmethoden ausgesetzt waren, einen statistisch betrachtet signifikanten Unterschied bezüglich der niedrigeren Bewusstheitsebene $(1,62,95 \%$ - CI $[2.77,0,46])$. Diejenigen Untersuchungsteilnehmer, die den expliziten Lehrmethoden ausgesetzt waren, zeigen hingegen einen statistisch signifikanten Unterschied bezüglich der höheren Bewusstheitsebene (8,77, 95\% - CI [7,73, 9,81]). Beim verzögerten Posttest zeigt 


\section{Ivana Šarić Šokčević: \\ Die Beziehung zwischen der Bewusstheit und den Lehrmethoden beim Er- lernen der (nicht) passivfähigen Verben im Deutschen als Fremdsprache}

sich in Bezug auf die höhere Bewusstheitsebene ein signifikanter Unterschied ( $p<$ ,01) zwischen denjenigen Untersuchungsteilnehmern, die den expliziten Lehrmethoden $(3,28,95 \%$ - CI $[2,68,3,88])$ ausgesetzt waren, und denjenigen Untersuchungsteilnehmern, die der impliziten Lehrmethode ausgesetzt waren.

Tabelle 7. Bonferroni-korrigierter post-hoc Test für die Variablen Bewusstheitsebene (niedrigere vs. höhere), Lehrmethode (explizit vs. implizit) und Test (unmittelbarer Posttest vs. verzögerter Posttest)

\begin{tabular}{|c|c|c|c|c|c|c|c|c|}
\hline \multirow[t]{2}{*}{ Test } & \multirow[t]{2}{*}{$\begin{array}{l}\text { Bewusstheits- } \\
\text { ebene }\end{array}$} & \multirow[t]{2}{*}{$\begin{array}{c}\text { (I) } \\
\text { explizit }\end{array}$} & \multirow[t]{2}{*}{$\begin{array}{c}(\mathrm{J}) \\
\text { implizit }\end{array}$} & \multirow[t]{2}{*}{$\begin{array}{l}\text { Mittlere } \\
\text { Diff. } \\
\text { (I-J) }\end{array}$} & \multirow[t]{2}{*}{$\begin{array}{l}\text { Standard- } \\
\text { fehler }\end{array}$} & \multirow[t]{2}{*}{ Sig. $^{b}$} & \multicolumn{2}{|c|}{$\begin{array}{c}\text { 95\% Konfidenzint. } \\
\text { für } \\
\text { die Differenz }{ }^{\mathrm{b}}\end{array}$} \\
\hline & & & & & & & Untergr. & Obergr. \\
\hline \multirow[t]{3}{*}{ unmittelbarer } & niedrigere & explizit & implizit & $-1,616^{*}$ & ,579 &, 006 & $-2,768$ &,- 464 \\
\hline & höhere & explizit & implizit & $8,768^{*}$ & ,522 & ,000 & 7,731 & 9,806 \\
\hline & niedrigere & explizit & implizit &, 437 & ,451 & 335 &,- 459 & 1,333 \\
\hline verzögerter & höhere & explizit & implizit & $3,280^{*}$ & ,300 &, 000 & 2,682 & 3,877 \\
\hline
\end{tabular}

Basiert auf den geschätzten Randmitteln; *. Die mittlere Differenz ist auf dem ,05-Niveau signifikant; b. Anpassung für die Mehrfachvergleiche: Bonferroni

Ferner wurde statistisch auch der zweiten Untersuchungsfrage nachgegangen. Tabelle 8 zeigt eine Interaktion zwischen den Variablen Test (unmittelbarer vs. verzögerter), Bewusstheitsebene (niedrigere vs. höhere) und Leistungsstufe (Fakultät vs. Gymnasium). Die verschiedenen Werte deuten auf eine statistische Relevanz der verschiedenen Variablen und Werte hin.

Tabelle 8. F-Werte der mixed ANOVA mit Messwiederholung mit Greenhouse-GeisserKorrektur für die Variablen Test (unmittelbarer Posttest vs. verzögerter Posttest), Bewusstheitsebene (niedrigere vs. höhere) und Leistungsstufe (Fakultät vs. Gymnasium)

\begin{tabular}{|l|r|r|r|}
\hline Variable & Df & \multicolumn{1}{|c|}{ F } & Partielles Eta-Quadrat \\
\hline Test & 1,87 & $72,999^{*}$ &, 456 \\
\hline Test*Leistungsstufe & 1,87 & 3,072 &, 034 \\
\hline Bewusstheitsebene*Leistungsstufe & 1,87 & $4,396^{*}$ &, 050 \\
\hline Test *Bewusstheitsebene*Leistungsstufe & 1,87 & $4,865^{*}$ &, 053 \\
\hline
\end{tabular}

Die Ergebnisse zeigen ebenfalls, dass die abhängige Variable Test statistisch betrachtet signifikant $\left(\mathrm{F}(1,87)=73,00, \mathrm{p}<, 01\right.$, partielles $\left.\varepsilon^{2}=, 46\right)$ ist, jedoch nicht in Interaktion mit der Variable Leistungsstufe steht. Der Bonferroni-korrigierte post-hoc Test für die Variablen Bewusstheitsebene (niedrigere vs. höhere) und Leistungsstufe (Fakultät vs. Gymnasium) (siehe Tabelle 9) zeigt eine schwache signifikante Interaktion $\left(\mathrm{F}(1,87)=4,40, \mathrm{p}<, 01\right.$, partielles $\left.\varepsilon^{2}=, 05\right)$ bezüglich der 
niedrigeren Bewusstheitsebene und der Variable Leistungsstufe (Fakultät vs. Gymnasium), wobei sich diese Interaktion auf die Untersuchungsgruppe der Germanistikstudenten bezieht.

Tabelle 9. Bonferroni-korrigierter post-hoc Test für die Variablen Bewusstheitsebene (niedrigere vs. höhere) und Leistungsstufe (Fakultät vs. Gymnasium)

\begin{tabular}{|c|c|c|c|c|c|c|c|}
\hline \multirow[t]{2}{*}{$\begin{array}{l}\text { Bewusstheits- } \\
\text { ebene }\end{array}$} & \multirow[t]{2}{*}{ (I) Leistungsst. } & \multirow[t]{2}{*}{ (J) Leistungsst. } & \multirow[t]{2}{*}{$\begin{array}{l}\text { Mittlere } \\
\text { Diff. } \\
\text { (I-J) }\end{array}$} & \multirow[t]{2}{*}{$\begin{array}{l}\text { Standard- } \\
\text { fehler }\end{array}$} & \multirow[t]{2}{*}{ Sig. $^{b}$} & \multicolumn{2}{|c|}{$\begin{array}{l}95 \% \text { Konfidenzin- } \\
\text { tervall für } \\
\text { die Differenz } \\
\end{array}$} \\
\hline & & & & & & Untergr. & Obergr. \\
\hline niedrigere & Fakultät & Gymnas. & $1,514^{*}$ & ,400 &, 000 &, 719 & 2,308 \\
\hline höhere & Fakultät & Gymnas. & ,175 & ,727 & 810 & $-1,270$ & 1,620 \\
\hline
\end{tabular}

Basiert auf den geschätzten Randmitteln; *. Die mittlere Differenz ist auf dem ,05-Niveau signifikant; b. Anpassung für die Mehrfachvergleiche: Bonferroni.

Die Interaktion der Variablen Test, Bewusstheitsebene und Leistungsstufe ist ebenfalls statistisch relevant $\left(\mathrm{F}(1,87)=4,87, \mathrm{p}<, 01\right.$, partielles $\left.\varepsilon^{2}=, 05\right)$. In diesem letzten Vergleich zwischen den Variablen Leistungsstufe (Fakultät vs. Gymnasium), Bewusstheitsebene (niedrigere vs. höhere) und Test (unmittelbarer vs. verzögerter Posttest) (siehe Tabelle 10) wird nur eine statistisch relevante Interaktion zwischen den Germanistikstudenten beim verzögerten Posttest und der niedrigeren Bewusstheitsebene sichtbar, jedoch nicht zwischen den anderen hier untersuchten Faktoren.

Tabelle 10. Bonferroni-korrigierter post-hoc Test für die Variablen Leistungsstufe (Fakultät vs. Gymnasium), Test (unmittelbarer vs. verzögerter Posttest) und Bewusstheitsebene (niedrigere vs. höhere)

\begin{tabular}{|c|c|c|c|c|c|c|c|c|}
\hline \multirow[t]{2}{*}{ Test } & \multirow[t]{2}{*}{$\begin{array}{l}\text { Bewusstheits- } \\
\text { ebene }\end{array}$} & \multirow[t]{2}{*}{$\begin{array}{l}\text { (I) Fa- } \\
\text { kultät }\end{array}$} & \multirow[t]{2}{*}{$\begin{array}{c}(\mathrm{J}) \\
\text { Gymnas. }\end{array}$} & \multirow[t]{2}{*}{$\begin{array}{l}\text { Mittlere } \\
\text { Diff. } \\
\text { (I-J) }\end{array}$} & \multirow[t]{2}{*}{$\begin{array}{l}\text { Standard- } \\
\text { fehler }\end{array}$} & \multirow[t]{2}{*}{ Sig. $^{b}$} & \multicolumn{2}{|c|}{$\begin{array}{l}\text { 95\% Konfidenzin- } \\
\text { tervall für } \\
\text { die Differenz } \\
\end{array}$} \\
\hline & & & & & & & Untergr. & Obergr. \\
\hline unmittelbarer & $\begin{array}{l}\text { niedrigere } \\
\text { höhere }\end{array}$ & $\begin{array}{l}\text { Fakultät } \\
\text { Fakultät }\end{array}$ & & $\begin{array}{l}, 534 \\
, 474\end{array}$ & $\begin{array}{r}, 602 \\
1,074\end{array}$ & $\begin{array}{l}378 \\
, 660\end{array}$ & $\begin{array}{r}-, 663 \\
-1,661\end{array}$ & $\begin{array}{l}1,731 \\
2,609\end{array}$ \\
\hline verzögerter & $\begin{array}{l}\text { niedrigere } \\
\text { höhere }\end{array}$ & $\begin{array}{l}\text { Fakultät } \\
\text { Fakultät }\end{array}$ & $\begin{array}{l}\text { Gymnas. } \\
\text { Gymnas }\end{array}$ & $\begin{array}{r}2,493^{*} \\
-, 124\end{array}$ & $\begin{array}{l}, 366 \\
, 462\end{array}$ & $\begin{array}{l}, 000 \\
, 790\end{array}$ & $\begin{array}{r}1,766 \\
-1,043\end{array}$ & $\begin{array}{r}3,221 \\
, 795\end{array}$ \\
\hline
\end{tabular}

Basiert auf den geschätzten Randmitteln; *. Die mittlere Differenz ist auf dem ,05-Niveau signifikant; b. Anpassung für die Mehrfachvergleiche: Bonferroni. 


\section{Diskussion}

Um die von Rod Ellis (2009) gestellte Forderung nach weiteren Untersuchungen, die sich mit den Mikroprozessen bei der Verarbeitung des Inputs bei Untersuchungsteilnehmern, die expliziten bzw. impliziten Lehrmethoden ausgesetzt waren, $\mathrm{zu}$ erfüllen, wurde in der vorliegenden Arbeit das Augenmerk auf die Beziehung zwischen den Bewusstheitsebenen und den Lernergebnissen der Untersuchungsteilnehmer nach der Anwendung der expliziten und impliziten Lehrmethoden gelegt. Zuerst werden die statistischen Daten bezüglich der ersten Untersuchungsfrage, die die Beziehung zwischen den Bewusstheitsebenen und der expliziten und impliziten Lehrmethode untersuchte, dargestellt. Die statistischen Resultate der vorliegenden Untersuchung zeigen in Bezug auf die erste Untersuchungsfrage, dass die Lernergebnisse beim unmittelbaren Posttest bei denjenigen Untersuchungsteilnehmern, die expliziten Lehrmethoden unterzogen wurden, besser waren als die Lernergebnisse bei denjenigen Untersuchungsteilnehmern, die impliziten Lehrmethoden ausgesetzt waren (vgl. Tabelle 3). Darüber hinaus konnte beim unmittelbaren Posttest eine starke Interaktion zwischen den Lernergebnissen der Untersuchungsteilnehmer, die der expliziten Lehrmethode unterzogen wurden, und der höheren Bewusstheitsebene (vgl. Tabelle 7) ermittelt werden. Es muss jedoch betont werden, dass die Think-aloud-Protokolle der Untersuchungsteilnehmer mit $[+\mathrm{N}]$ für die niedrigere Bewusstheitsebene oder $[+\mathrm{U}]$ für die höhere Bewusstheitsebene kodifiziert wurden. Zieht man jedoch in Betracht, dass diejenigen Untersuchungsteilnehmer, die mit $[+\mathrm{U}]$ (höhere Bewusstheitsebene, die Ebene des Understanding) kodifiziert wurden, gleichzeitig auch mit $[+\mathrm{N}]$ (niedrigere Bewusstheitsebene, die Ebene des Noticing) hätten kodifiziert werden können, da das Erreichen der höheren Bewusstheitsebene durch das Bemerken, die niedrigere Bewusstheitsebene gewährleistet ist, kann man davon ausgehen, dass diese Teilnehmer eine starke Interaktion zwischen den Lernergebnissen beim unmittelbaren Posttest derjenigen Untersuchungsteilnehmer, die expliziten Lehrmethoden ausgesetzt waren, sowohl bei der niedrigeren als auch der höheren Bewusstheitsebene aufzeigten. Im Gegensatz dazu wurde beim unmittelbaren Posttest bei denjenigen Untersuchungsteilnehmern, die der impliziten Lehrmethode ausgesetzt waren, nur eine Interaktion zwischen den Lernergebnissen und der niedrigeren Bewusstheitsebene festgestellt, jedoch keine signifikante Interaktion mit der höheren Bewusstheitsebene.

Diese Untersuchungsergebnisse bestätigen Leows empirische Daten (1997) in Bezug auf die Erforschung der Bewusstheitsebenen in der Input-intake-Phase. Anhand dieser Daten schlussfolgerte Leow (ebd.), dass die Bewusstheit eine fördernde Rolle im fremdsprachlichen Lernprozess hat, wobei die metalinguistische Bewusstheit, die sich in der höheren Bewusstheitsebene widerspiegelt, in Interakti- 
on mit den besseren Lernergebnissen bei den Posttests steht. Leow $(1997 ; 2015)$ geht deshalb davon aus, dass mehr Bewusstheit in mehr Wiedererkennung (engl. recognition) und dann auch einer genaueren Produktion der bemerkten Formen resultiert, wobei diese dann die weitere Verarbeitung der Zielstruktur fördert. Die Ergebnisse der vorliegenden Untersuchung sind ebenfalls in Einklang mit diesen Annahmen, da sowohl diejenigen Untersuchungsteilnehmer, die der expliziten als auch diejenigen, die der impliziten Lehrmethode ausgesetzt waren, statistisch ihre Lernergebnisse vom Pretest zum unmittelbaren Posttest verbessert haben (vgl. Tabelle 3). Es zeichnet sich außerdem eine statistisch signifikante Interaktion sowohl zwischen der niedrigeren als auch der höheren Bewusstheitsebene und den Lernergebnissen beim unmittelbaren Posttest (Tabelle 7) ab. Man kann daher wie Leow (1997; 2015) davon ausgehen, dass die Bewusstheit eine fördernde Rolle im fremdsprachlichen Lernprozess hat, wobei die verschiedenen Bewusstheitsebenen die Art und Weise, wie Lernmaterial verarbeitet wird, beeinflussen können (Leow 1997: 188). Es kann geschlussfolgert werden, dass die höhere Bewusstheitsebene die Verarbeitung der zielsprachlichen komplexen Struktur beim unmittelbaren Posttest stärker als die niedrigere Bewusstheitsebene fördert.

Die qualitativen und statistischen Ergebnisse der vorliegenden Untersuchung stimmen auch mit den Untersuchungsergebnissen von Rosa und O'Neill (1999) überein, die eine starke Korrelation zwischen der höheren Bewusstheitsebene und den Lernergebnissen beim unmittelbaren Posttest bestätigen konnten, und daraus schlussfolgerten, dass die Bewusstheit und die Leistung in einem proportionalen Verhältnis zueinander stehen.

Darüber hinaus konnten auch Parallelen zwischen der vorliegenden Untersuchung und den Untersuchungsergebnissen von Rosa und Leow (2004) festgestellt werden. In der Untersuchung von Rosa und Leow (2004) wurde ebenfalls eine Korrelation zwischen den besseren Lernergebnissen und den Bewusstheitsebenen errechnet. Zum einen deuten ihre Untersuchungsergebnisse darauf hin, dass schon die niedrigere Bewusstheitsebene ausreicht, um ein besseres Lernergebnis vom Pretest zum unmittelbaren Posttest zu erzielen, da die niedrigere Bewusstheitsebene mit den Lernergebnissen der impliziten Untersuchungsgruppe beim unmittelbaren Posttest in Korrelation stand. Dies konnte auch in der vorliegenden Untersuchung beobachtet werden (vgl. Tabelle 7). Diejenigen Untersuchungsteilnehmer, die impliziten Lehrmethoden ausgesetzt waren, zeigten beim unmittelbaren Posttest eine statistisch betrachtet signifikante Interaktion mit der niedrigeren Bewusstheitsebene, was sich ebenfalls mit den Ergebnissen von Rosa und Leow (ebd.) deckt. Darüber hinaus haben auch diejenigen Untersuchungsteilnehmer, die der impliziten Lehrmethode ausgesetzt waren, einen signifikanten Unterschied vom Pretest zum 
unmittelbaren Posttest gezeigt (Tabelle 3). Auch dies ist bezüglich der Interaktion zwischen der impliziten Lehrmethode, den Lernergebnissen vom Pretest zum unmittelbaren Posttest und der niedrigeren Bewusstheitsebene in Einklang mit den Resultaten von Rosa und Leow (ebd.). Dies lässt die Schlussfolgerung zu, dass sowohl die niedrigere Bewusstheitsebene als auch die höhere Bewusstheitsebene mit der Leistung beim unmittelbaren Posttest in Korrelation steht. Darüber hinaus haben Rosa und Leow (ebd.) mit ihren Untersuchungsergebnisse bestätigt, dass es eine starke positive Korrelation zwischen der höheren Bewusstheitsebene und den Lernergebnissen beim unmittelbaren Posttest gibt. Dies konnte auch in der vorliegenden Untersuchung bestätigt werden, da die höhere Bewusstheitsebene auch in der vorliegenden Untersuchung in starker Interaktion mit den besseren Lernergebnissen beim unmittelbaren Posttest steht (vgl. Tabelle 6). Diese Untersuchungsergebnisse konnten bislang auch in anderen ähnlichen Untersuchungen (z. B. Medina 2015; de la Fuente 2015) bestätigt werden.

Zusammenfassend kann man anhand der empirischen Daten der vorliegenden Untersuchung bezüglich der niedrigeren Bewusstheitsebene schlussfolgern, dass die Bewusstheit in der frühen Input-intake-Phase eine fördernde und unterscheidende Rolle zu spielen scheint. Die höhere Bewusstheitsebene steht jedoch in Interaktion mit den besseren Lernergebnissen. Diese Schlussfolgerung kann natürlich auch als zusätzliche Bestätigung von Schmidts Noticing-Hypothese (1990; 1993; 1995) betrachtet werden, da er der Auffassung ist, dass mehr Bewusstheit zu mehr Intake führt (d. h. zu besseren Lernresultaten), aber auch, dass Input ohne jegliche Bewusstheit nicht in Intake verwandelt werden kann.

Relevant und interessant sind auch die erhaltenen Untersuchungsergebnisse bezüglich des verzögerten Posttests. Es konnte ein signifikanter Unterschied beim verzögerten Posttest zugunsten der expliziten Lehrmethode (Tabelle 7) bezüglich der höheren Bewusstheitsebene festgehalten werden. Diese Ergebnisse deuten darauf hin, dass die besseren Lernergebnisse nach der Anwendung der expliziten Lehrmethode und dem Erreichen der höheren Bewusstheitsebene in starker Interaktion stehen. Die Beziehung, die zwischen der niedrigeren Bewusstheitsebene beim unmittelbaren Posttest festgehalten wurde, konnte beim verzögerten Posttest nicht bestätigt werden. Es ist nur ein signifikanter Unterschied bezüglich der Interaktion zwischen der expliziten Lehrmethode und der höheren Bewusstheitsebene erhalten geblieben. Eine mögliche Erklärung ist die Tatsache, dass einige Fremd- und Zweitsprachenforscher (z. B. Schmidt 1990; Robinson 1995; Leow 2001, 2015) annehmen, dass nur die höhere Bewusstheitsebene langfristig zum Erlernen der Zielstruktur führt. Bei denjenigen Untersuchungsteilnehmern, bei denen die höhere Bewusstheitsebene, die Ebene des Verstehens, erreicht werden konnte, wurde nicht 


\section{EZIKOSLOVLJE \\ 22.1 (2021): 99-148}

nur die Aufmerksamkeit der Untersuchungsteilnehmer auf das Prozessieren des Inputs in Intake gelenkt, sondern es ist ihnen auch gelungen, die zugrunde liegende Regel bezüglich der fremdsprachlichen Zielstruktur zu verstehen und laut zu artikulieren. Man kann schlussfolgern, dass je höher die Bewusstheit über eine bestimmte Zielstruktur ist, desto effektiver diese in den unterschiedlichen Phasen des fremdsprachlichen Lernprozesses verarbeitet und somit auch am Ende erlernt werden kann.

Einen wichtigen Aspekt scheint die Lehrmethode darzustellen, da die Instruktionsverfahren, die sich auf dem Kontinuum an die explizite Lehrmethode anlehnen, sich in diesem Prozess als besonders effektiv gezeigt haben. Dies deckt sich auch mit anderen theoretischen Auslegungen (Schmidt 1990, 1994, 1995; Robinson 1995; Leow 1997, 2001, 2015) und dementsprechenden empirischen Untersuchungsergebnissen (Alanen 1995; Leow 1998; Rosa und O'Neill 1999; Bagarić 2001; Rosa und Leow 2004; Radwan 2005).

Die Ergebnisse der vorliegenden Untersuchung bestätigen somit Radwans Annahmen (2005), die auf die Ergebnisse von Robinson (1997) zurückgehen, und sich auf seine empirischen Daten stützen, da eine starke positive Korrelation zwischen den besseren Lernergebnissen, der höheren Bewusstheitsebene und der expliziten Lehrmethode sowohl beim unmittelbaren als auch verzögerten Posttest nachgewiesen werden konnte. Somit konnte auch die Hypothese in Bezug auf die erste Untersuchungsfrage bestätigt werden, da die Lernergebnisse des unmittelbaren und verzögerten Posttests bei denjenigen Teilnehmern, die expliziten Lehrmethoden ausgesetzt waren, eine stärkere Interaktion bezüglich der Bewusstheitsebenen zeigen als bei denjenigen Teilnehmern, die impliziten Lehrmethoden unterzogen wurden.

In der vorliegenden Untersuchung wurde mit der zweiten Untersuchungsfrage auf Radwans (2005) und Calderóns (2013) Forderungen nach weiteren Untersuchungen bezüglich des Einflusses des Sprachkenntnisstandes auf die Bewusstheitsvorgänge eingegangen. Es wurde demnach die Beziehung zwischen den Lernergebnissen der Posttests nach der Anwendung der expliziten und impliziten Lehrmethoden, den Bewusstheitsebenen und dem Sprachkenntnisstand ${ }^{10}$ weiter zu beleuchten versucht. Calderón (2013) ging einer ähnlichen Frage in ihrer Untersuchung nach und konnte unter anderem Unterschiede zwischen den verschiedenen Sprachkenntnisständen und den beiden Bewusstheitsebenen nachweisen, wobei sich dieser Unterschied jedoch nicht auch statistisch bei den Lernergebnissen zeigte. Diejenigen Untersuchungsteilnehmer, die sich auf einem höheren Sprachken-

${ }^{10}$ Aus Vereinfachungsgründen wurde der Sprachkenntnisstand bei der statistischen Analyse (Kapitel 4.1.) mit der Variable Leistungsstufe beschrieben. 
Ivana Šarić Šokčević:

Die Beziehung zwischen der Bewusstheit und den Lehrmethoden beim Erlernen der (nicht) passivfähigen Verben im Deutschen als Fremdsprache

ntnisstand befanden, zeigten in Calderóns (ebd.) Untersuchung mehr Bewusstheit sowohl auf der niedrigeren als auch der höheren Bewusstheitsebene als diejenigen Untersuchungsteilnehmer, die sich auf einem niedrigeren Sprachkenntnisstand befanden. In der vorliegenden Untersuchung ging man von der Hypothese aus, dass die Lernergebnisse der Germanistikstudenten (höherer Sprachkenntnisstand, größeres Vorwissen bezüglich der Zielstruktur) beim unmittelbaren und verzögerten Posttest, unabhängig von der Art der Lehrmethode, eine Interaktion sowohl mit der niedrigeren als auch höheren Bewusstheitsebene aufweisen werden im Gegensatz zu den Lernergebnissen der Gymnasiasten (niedrigerer Sprachkenntnisstand, weniger sprachliches Vorwissen bezüglich der Zielstruktur). Man geht davon aus, dass die Germanistikstudenten auf Grund der Tatsache, dass sie sich schon länger, intensiver, sowohl explizit als auch implizit mit dem Deutschen beschäftigen und motivierter für die Aneignung neuer Strukturen sind als die Gymnasiasten, schneller und effektiver die beiden Bewusstheitsebenen erreichen werden, was grundsätzlich auch zu besseren Lernleistungen führen könnte. Die statistischen Resultate jedoch konnten diese zweite Hypothese nur teilweise bestätigen.

Die ANOVA-Analyse zeigt eine Interaktion nur zwischen der niedrigeren Bewusstheitsebene und den Lernergebnissen beim verzögerten Posttest bei den Germanistikstudenten (siehe Tabelle 10). Eine solche Interaktion konnte beim unmittelbaren Posttest nicht nachgewiesen werden, obwohl beim unmittelbaren Posttest insgesamt sehr viel bessere Lernresultate als beim verzögerten Posttest erzielt wurden. Es konnte keine Interaktion zwischen dem Sprachkenntnisstand, der höheren Bewusstheitsebene und der Lernergebnisse festgestellt werden.

Der Sprachkenntnisstand scheint offensichtlich erst beim verzögerten Posttest eine Rolle zu spielen und nicht, wie in unserer Hypothese angenommen, auch beim unmittelbaren Posttest. Dies lässt die Schlussfolgerungen zu, dass der Sprachkenntnisstand nur bedingt auf die Bewusstheitsebenen und die Lernleistung einflussreich sein kann. Obwohl die Germanistikstudenten über ein besseres explizites und implizites Sprachwissen verfügen, konnten sie diesen Vorteil erst beim verzögerten Posttest ausnutzen. Der Sprachkenntnisstand scheint keine Rolle beim unmittelbaren Posttest zu spielen, aber nachdem die Lernenden die Zielstruktur aufgenommen haben, kann der Sprachkenntnisstand beim verzögerten Posttest wichtig sein, was sich in der Interaktion zwischen der niedrigeren Bewusstheitsebene und den Lernergebnissen beim verzögerten Posttest bei den Gymnasiasten widerspiegelt. Es ist möglich, dass sich die Germanistikstudenten beim verzögerten Posttest des Sprachbewusstseins in Form des bemerkten Inputs bedient haben, wobei somit die niedrigere Bewusstheitsebene beim Lösen der Aufgaben aktiviert wurde. Im Gegensatz dazu konnte jedoch nicht die höhere Bewusstheitsebene aktiviert 


\section{JEZIKOSLOVLJE \\ 22.1 (2021): 99-148}

werden, da die zielsprachliche komplexe Struktur nicht ausreichend ins Intake aufgenommen wurde. Leow (2015: 2020) nimmt an, dass dies auch eine Erklärung für die Untersuchungsergebnisse von Calderón ist, da auch in ihrer Untersuchung eine komplexe Zielstruktur (der Konjunktiv Plusquamperfekt im Englischen) als Untersuchungsgegenstand diente. Da auch in der vorliegenden Untersuchung eine komplexe, mehrdimensionale fremdsprachliche Zielstruktur verwendet wurde, darf dieser mögliche Einfluss nicht außer Acht gelassen werden. Es wurden diesbezüglich auch Beobachtungen beim Transkribieren der Think-aloud Protokolle und der qualitativen Auswertung dieser Daten gemacht. Diese Beobachtungen beziehen sich besonders auf die zumeist gescheiterten Versuche des Verstehens der komplexen Zielstruktur während der Lösung des verzögerten Posttests bei den Gymnasiasten, aber auch den Germanistikstudenten, die eine schwächere Lernleistung sowohl beim unmittelbaren als auch verspäteten Posttest zeigten. Diejenigen Untersuchungsteilnehmer, die sich auf einem höheren Sprachkenntnisstand befinden, scheinen mit einem gewissen Zeitabstand hinsichtlich der Bearbeitung der Zielstruktur mithilfe ihres Vorwissens, eine bessere Voraussetzung für das Bemerken dieser Zielstruktur zu haben. Dies bezieht sich jedoch nur auf das Bemerken, nicht auch auf das Verstehen, das sich in der höheren Bewusstheitsebene widerspiegelt, aber auch nach den bisherigen Erkenntnissen in Interaktion mit besseren Lernergebnissen zu stehen scheint.

Eine mögliche Erklärung für die unterschiedlichen Untersuchungsergebnisse in der vorliegenden und der von Calderón (2013) durchgeführten Untersuchung, könnte in der Tatsache liegen, dass die Untersuchungsteilnehmer von Calderón (ebd.) englische Studenten des ersten oder dritten Semesters eines Spanischstudiums waren. Der Unterschied in Bezug auf den Sprachkenntnisstand kommt daher wahrscheinlich nicht so stark zum Vorschein, wie etwa zwischen den Untersuchungsteilnehmern in der vorliegenden Untersuchung. Darüber hinaus scheint auch der Aspekt der Motivation in Calderóns Untersuchung (ebd.) nicht so maßgebend wie in der vorliegenden Untersuchung. Es ist anzunehmen, dass die Studenten, die sich selbstständig eigenwillig zu einem fremdsprachlichen Studium entschieden haben, motivierter sind. Ferner scheint auch der generelle Vergleich der Untersuchungsergebnisse schwierig und unzureichend objektiv zu sein, da sich die Untersuchungsdesigns von Calderón (ebd.) und der vorliegenden Untersuchung, z. B. hinsichtlich der Aspekte Untersuchungsinstrumente, Anzahl der Untersuchungsteilnehmer, Dauer der Untersuchung, zu deutlich unterscheiden. Genau dies, der Mangel an vergleichbaren Untersuchungen, besonders im methodologischen Bereich, wurde auch von Leow (2015) mehrfach thematisiert.

Man kann schlussfolgern, dass die Lernergebnisse des verzögerten Posttests bei 
Ivana Šarić Šokčević:

Die Beziehung zwischen der Bewusstheit und den Lehrmethoden beim Erlernen der (nicht) passivfähigen Verben im Deutschen als Fremdsprache

denjenigen Teilnehmern, die sich auf einem höheren Sprachkenntnisstand befinden und über ein größeres Vorwissen verfügen, nur eine Interaktion mit der niedrigeren Bewusstheitsebene aufweisen. Man kann annehmen, dass der Sprachkenntnisstand aufgrund des zugrunde liegenden expliziten und impliziten Sprachwissens nur bedingt einflussreich sein kann. Dieser Einfluss scheint jedoch beim Verstehen und damit Erlernen der vorliegenden komplexen Zielstruktur keine ausschlaggebende Rolle zu spielen, wie beispielsweise dies bei der Lehrmethode der Fall ist.

\section{Einschränkungen und Ausblick}

Leow (2015) setzt sich besonders für die kritische Auseinandersetzung mit der Methodologie und den Einschränkungen der Untersuchungen bezüglich der Bewusstheit ein. Eine der Einschränkungen der vorliegenden Untersuchung bezieht sich auf die Think-aloud-Methode. In dieser Arbeit wurden offline Think-aloudProtokolle zur Datenerhebung bezüglich der Bewusstheitsebenen herangezogen. Dies könnte Fragen bezüglich der internen Validität der Untersuchung aufwerfen, da nicht der Prozess, das Verarbeiten der Daten beim Untersuchungsteilnehmer während des Treatments gemessen wurde, sondern das Produkt, das nach dieser Verarbeitungsphase zustande kommt. Aufgrund des Kontextes und der spezifischen Eigenschaften der Untersuchungsteilnehmer war eine solche Anwendung der Think-aloud-Methode notwendig. Man ist davon ausgegangen, dass online Thinkaloud-Protokolle eine kognitiv zu extreme Untersuchungsumgebung für die Teilnehmer darstellen würde, was z. B. zu einem Reaktivitätseffekt hätte führen können. Nimmt man zusätzlich noch in Betracht, dass es um ein Experiment geht, das im Klassenzimmer während des Unterrichts durchgeführt wurde, war dies ferner ein zusätzlicher Grund, weshalb man sich für offline Protokolle entscheiden musste. Der Einsatz von online Think-aloud-Protokollen hätte sich in einem solchen Experiment noch schwieriger gestaltet und womöglich die interne Validität der Untersuchung vollkommen in Frage stellen können. Es dürfen auch die möglichen spezifischen Merkmale der Untersuchungsteilnehmer nicht außer Acht gelassen werden. Es ist aufgrund des Lernkontextes davon auszugehen, dass einige Untersuchungsteilnehmer eventuell aus individuellen Gründen nicht bereit gewesen sein könnten, ihre Gedanken während des Unterrichts laut zu artikulieren. Dafür kann es einige Gründe geben: kognitive Überforderung (gleichzeitiges Verbalisieren von Gedanken und aktiver Unterricht), Introvertiertheit als Charaktermerkmal, erstmalige Beteiligung an einer sprachwissenschaftlichen Untersuchung usw. In Zukunft können solche Bedenken minimalisiert werden, indem man beispielsweise den Schwerpunkt auf die Verlängerung der Vorbereitungsphase auf die Think-aloud- 
Methode legt. In der vorliegenden Untersuchung betrug diese nur eine Schulstunde. Darüber hinaus scheint auch eine der Möglichkeiten zu sein, zusätzliche Interviews zur Klärung bestimmter für den Untersuchungsleiter nicht erforschbarer Hindernisse, auf die man während der Transkriptionsphase gestoßen ist, einzusetzen. Ferner ist auch die Anwendung der Eye-Tracking-Methode (z.B. Andringa 2020; Sachs, Hamrick, McCormick und Leow 2020) besonders ratsam, um einen besseren Einblick in die Bewusstheitsebenen zu bekommen.

Letztendlich müssen auch einige Bedenken über die Deskriptoren bezüglich der Bewusstheitsebenen geäußert werden. In der vorliegenden Untersuchung entschied man sich in Anlehnung an ähnliche Untersuchungen (z. B. Schmidt 1990, 1993, 1994, 1995; Rosa und O'Neill 1999; Rosa und Leow 2004; Sachs und Suh 2007) die Bewusstheitsebenen in zwei Kategorien einzuteilen: die niedrigere und die höhere Bewusstheitsebene. Die qualitative Analyse der vorliegenden Untersuchung hat jedoch gezeigt, dass ein Bedarf an einer weiteren Ausdifferenzierung zwischen den Bewusstheitsebenen besteht, ähnlich wie bei Leow (1997) und Bagarić (2001).

\section{Schlussfolgerung und pädagogische Implikationen}

Die vorliegende Untersuchung kann aufschlussreiche qualitative und quantitative Daten bezüglich der Beziehung zwischen der Bewusstheit und den expliziten und implizite Lehrmethoden beim Erlernen der (nicht) passivfähigen Verben im Deutschen als Fremdsprache aufzeigen. In dieser Beziehung sollte vor allem die fördernde und unterscheidende Rolle der Bewusstheit beim Erlernen der komplexen Zielstruktur hervorgehoben werden, wobei sich auch die Lehrmethoden als einflussreicher Faktor erwiesen haben. Auf eine effektivere und gesteigerte Leistung der Untersuchungsteilnehmer vom Pretest zum verzögerten Posttest haben offensichtlich verschiedene Faktoren Einfluss. Die explizite Lernmethode und die Bewusstheitsebenen scheinen eine entscheidende und unterscheidende Rolle in der Umwandlung von Input in Intake spielen. Die implizite Lehrmethode kann zwar auch zur Förderung der niedrigeren Bewusstheitsebene beitragen, jedoch hat sich die explizite Lehrmethode am effektivsten bei der Förderung der höheren Bewusstheitsebene erwiesen, was insgesamt zu besseren Lernleistungen geführt hat. Es besteht ferner bei den Germanistikstudenten eine Interaktion zwischen den Lernergebnissen und der niedrigeren Bewusstheitsebene, jedoch nicht auch für die höhere Bewusstheitsebene.

Da jede psycholinguistische Untersuchung im Zweit- und Fremdsprachenerwerbsbereich die pädagogische Anwendung der theoretischen und empirisch gewonnen 
Daten zum Ziel haben sollte, wird an dieser Stelle versucht, einige pädagogische Schlussfolgerungen bezüglich der in der vorliegenden Untersuchung gewonnen Daten zu ziehen:

1. Da sich die explizite Lehrmethode bei der vorliegenden komplexen, mehrdimensionalen fremdsprachlichen Zielstruktur als insgesamt effektiver zeigte, könnte sie vor allem in den frühen Phasen des fremdsprachlichen Lernprozesses eingesetzt werden. Auf diese Weise könnten die Bewusstheitsebenen (schneller) aktiviert werden, was eine effektivere und langfristig robustere Umwandlung des Inputs in Intake gewährleisten könnte. Darüber hinaus könnte dies dann auch die Lernenden zur weiteren Verarbeitung des Intakes und der Hypothesenbildung bezüglich der Zielstruktur motivieren. Da nach der Input-intake-Phase im fremdsprachlichen Lernprozess eine Einübungsphase folgt, wird eine solche Anwendung auch von Vorteil für die weitere Verarbeitung des Inputs in Output sein.

2. Bessere Lernleistungen wurden beim unmittelbaren Posttest sowohl von denjenigen Untersuchungsteilnehmern, die expliziten Instruktionsmaßnamen als auch von denjenigen, die impliziten Instruktionsmaßnahmen ausgesetzt waren, erzielt. Vor allem für schwächere Lernende könnte es vorteilhaft sein sich verschiedener expliziter Instruktionsmaßnahmen, die sich auf dem explizit-impliziten Kontinuum befinden, zu bedienen. Da auch die implizite Lehrmethode zu einer positiven wenn auch geringeren Lernleistung führte, könnte die Zielstruktur vielleicht zuerst implizit eingesetzt werden, um zuerst die niedrigere Bewusstheitsebene zu aktivieren, und um dann später mithilfe der expliziten Lehrmethode, die höhere Bewusstheitsebene zu erreichen, und somit die Weiterverarbeitung der Zielstruktur im fremdsprachlichen Lernprozess anzukurbeln. Dies könnte für die schwächeren Lernenden vorteilhafter sein, da sie kognitiv nicht zu Beginn der Input-intake-Phase überfordert sein würden und die Möglichkeit erhalten würden, langsam die komplexe Zielstruktur aufzunehmen, dann in ihre Bestandteile zu zerlegen und sie somit besser zu erfassen.

Am Ende muss nochmals betont werden, dass es einen weiteren Bedarf an ähnlichen Untersuchungen mit einer größeren Anzahl von Untersuchungsteilnehmern gibt, um die bereits in Laboruntersuchungen überprüften Hypothesen und empirischen Ergebnisse im schulischen Lernkontext, im Klassenzimmer, zu erforschen, um aus diesen Erkenntnissen bessere pädagogische Implikationen erschließen zu können. 


\section{Literatur}

Alanen, Riika. 1995. Input enhancement and rule presentation in second language acquisition. In Schmidt, Richard W. (Hrsg.), Attention and awareness in foreign language learning Honolulu, 259-302. Honolulu, HI: University of Hawaii Press.

Alderson, Charles; Clapham, Caroline; Steel, David. 1997. Metalinguistic knowledge, language aptitude and language proficiency. Language Teaching Research 1(2). 93-121. https://doi.org/10.1177/136216889700100202

Anderson, John R. 1982. Acquisition of cognitive skill. Psychological Review 89(4). 369406. http://dx.doi.org/10.1037/0033-295X.89.4.369

Andringa, Sible. 2020. The emergence of awareness in uninstructed L2 learning: A visual world eye tracking study. Second Language Research 36(3). 335-357. https://doi.org/10.1177/0267658320915502

Ammar, Ahlem. 2008. Prompts and recasts: Differential effects on second language morphosyntax. Language Teaching Research 12(2). 183-210. https://doi.org/10.1177/1362168807086287

Andrews, Stephen. 2007. Researching and developing teacher language awareness: Developments and future directions. In Cummins, Jim; Davison, Chris (Hrsg.), International Handbook of English Language Teaching, 945-959. New York: Springer.

Bagarić, Vesna. 2001. Odnos jezične svjesnosti i uspjeha učenika u engleskom kao stranom jeziku. Unveröffentlichte Magisterarbeit. Zagreb: Philosophische Fakulät.

Benati, Alessandro. 2005. The effects of processing instruction, traditional instruction and meaning - output instruction on the acquisition of the English Past Simple tense. Language Teaching Research 9(1). 67-93. https://doi.org/10.1191/13621688051r154oa

Calderón, Anne Maria. 2013. The effects of L2 learner proficiency on depth of processing, levels of awareness, and intake. In Bergsleithner, Joara Martin; Frota, Sylvia Nagem; Yoshioka, Jim Kei (Hrsg.), Noticing and second language acquisition: Studies in honor of Richard Schmidt, 103-121. Honolulu: University of Hawai'i, National Foreign Language Resource Center.

Carroll, Susanne; Swain, Merrill. 1993. Explicit and implicit negative feedback: An empirical study of the learning of linguistic generalization. Studies in Second Language Acquisition 15. 357-386. http://dx.doi.org/10.1017/S0272263100012158

Chan, Rick; Leung, Janny. 2014. Implicit learning of L2 word stress rules. Second Language Research 2014 30(4). 463-484. https://doi.org/10.1177/0267658313510169

Cohen, Jacob. 1988. Set correlation and contingency tables. Applied Psychological Measurements 12(4). 425-434. https://doi.org/10.1177/014662168801200410

De Graaff, Rick. 1997. The eXperanto experiment: Effects of explicit instruction on second language acquisition. Studies in Second Language Acquisition 19. 249-276. http://dx.doi.org/10.1017/S0272263197002064 
DeKeyser, Robert M. 1993. The effect of error correction on L2 grammar knowledge and oral proficiency. Modern Language Journal 77(4). 501-514. https://doi.org/10.1111/j.1540-4781.1993.tb01999.x

DeKeyser, Robert M. 1995. Learning L2 grammar rules. Studies in Second Language Acquisition 17(3). 379-410.

DeKeyser, Robert M. 1997. Beyond explicit rule larning: Automatizing second language morphosyntax. Studies in Second Language Acquisition 19(2). 196-221.

DeKeyser, Robert M. 2003. Implicit and explicit learning. In Doughty, Catherine J.; Long, Michael H. (Hrsg.), The handbook of second language acquisition, 313-348. New York: Wiley-Blackwell. http://dx.doi.org/10.1002/9780470756492.ch11

De la Fuente, Maria J. 2015. Explicit corrective feedback and computer-based, formfocused instruction: The role of L1 in promoting a of L2 forms. In Leow, Ronald P.; Cerezo, Luis; Baralt, Melissa (Hrsg.), A Psycholinguistic approach to technology and language learning, 171-198. Berlin: De Gruyter Mouton. http://dx.doi.org/10.1515/9781614513674-012

Doughty, Catherine. 1991. Second language acquisition does make a difference: Evidence from an empirical study of SL relativization. Studies in Second Language Acquisition 13 (3). 431-469.

Doughty, Catherine; Williams, Jessica. 1998. Focus on form in classroom second language acquisition. New York: Cambridge University Press.

Elder, Catherine; Manwaring, Diane. 2004. The relationship between metalinguistic knowledge and learning outcomes among undergraduate students of Chinese. Language Awareness 13(3). 145-162. http://dx.doi.org/10.1080/09658410408667092

Ellis, Rod. 1995. Interpretation tasks for grammar teaching. TESOL Quarterly 29(1). 87107. http://dx.doi.org/10.2307/3587806

Ellis, Rod. 2000. Task-based research and language pedagogy. Language Teaching Research 4(3). 193-220. https://doi.org/10.1177/136216880000400302

Ellis, Rod. 2005. Principles of instructed language learning. System 33(2). 209-224. https://doi.org/10.1016/j.system.2004.12.006

Ellis, Rod. 2009. Task-based language teaching: Sorting out the misunderstandings. International Journal of Applied Linguistics 19(3). 221-246. http://doi.org/10.1111/j.14734192.2009.00231.x

Ellis, Rod. 2015. Understanding second language acquisition. Second edition. Oxford: Oxford University Press. http://dx.doi.org/10.1016/j.system.2017.07.013

Ellis, Rod; Loewen, Shawn; Erlam, Rosemary. 2006. Implicit and explicit corrective feedback and the acquisition of L2 grammar. Studies in Second Language Acquisition 28(2). 339-368. https://doi.org/10.1017/S0272263106060141

Faretta-Stutenberg, Mandy; Morgan-Short, Kara. 2011. Learning without awareness reconsidered: A replication of Williams (2005). In Granena, Gisela; Koeth, Joel; Lee-Ellis, 
Sunyoung; Lukyachenko, Anna; Prieto Botana, Goretti; Rhoades, Elizabeth (Hrsg.), Selected proceeedings of the 2010 Second Language Research Forum: Reconsidering SLA research, dimensions and dierctios, 18-28. Somerville: Cascadilla Proceddings Project.

Fotos, Sandra. 1993. Consciousness raising and noticing through focus on form: Grammar task performance versus formal instruction. Applied Linguistics 14(4). 385-407. https://doi.org/10.1093/applin/14.4.385

Gass, Susan M.; Mackey, Alison; Pica, Teresa. 1998. The role of input and interaction in second language acquisition: An introduction. Modern Language Journal 82(3). 299307. https://doi.org/10.1111/j.1540-4781.1998.tb01206.x

Gass, Susan M.; Svetics, Ildiko; Lemelin, Sarah. 2003. Differential effects of attention. Language Learning 53(3). 497-545. https://doi.org/10.1111/1467-9922.00233

Goo, Jaemyung; Granena, Gisela; Yilmaz, Yucel; Novella, Miguel. 2015. Implicit and explicit instruction in L2 learning. In Rebuschat, Patrick (Hrsg.), Implicit and explicit learning of languages, 443-482. Amsterdam: John Benjamins. https://doi.org/10.1075/sibil.48.18goo

Green, Peter S.; Hecht, Karlheinz. 1992. Implicit and explicit grammar: An empirical study. Applied Linguistics 13(2). 160-184.

Han, Youngju; Ellis, Rod. 1998. Implicit knowledge, explicit knowledge and general language proficiency. Language Teaching Research 2(1). 1-23. https://doi.org/10.1177/136216889800200102

Hatch, Evelyn; Lazaraton, Anne. 1991. The research manual: Design and statistics for applied linguistics. Boston: Heinle \& Heinle.

Hulstijn, Jan H.; Hollander, Merel; Greidanus, Tine. 1996. Incidental vocabulary learning by advanced foreign language students: The influence of marginal glosses, dictionary use, and reoccurrence of unknown words. The Modern Language Journal 80(3). 327339. https://doi.org/10.2307/329439

Leow, Ronald P. 1997. Attention, awareness, and foreign language behavior. Language Learning 47(3). 467-506. https://doi.org/10.1111/0023-8333.00017

Leow, Ronald P. 1998. The effects of amount and type of exposure on adult learners' L2 development in SLA. Modern Language Journal 82(1). 49-68. https://doi.org/10.2307/328683

Leow, Ronald P. 2000. A study of the role of awareness in foreign language behavior: Aware versus unaware learners. Studies in Second Language Acquisition 22(4). 557584.

Leow, Ronald P. 2001. Attention, awareness and foreign language behavior. Language Learning 51(1). 113-155. https://doi.org/10.1111/j.1467-1770.2001.tb00016.x

Leow, Ronald P. 2012. Attention, noticing, and awareness in second language acquisition. 
In Chapelle, Carol A. (Hrsg.), The Encyclopedia of Applied Linguistics, 1-7. Oxford: Wiley-Blackwell. https://doi.org/10.1002/9781405198431.wbeal0058

Leow, Ronald P. 2015. Explicit learning in the L2 classroom: A student-centered approach. London: Routledge. https://doi.org/10.4324/9781315887074

Leow, Ronald P.; Johnson, Ellen; Zárate-Sández, Germàn. 2011. Getting a grip on the slippery construct of awareness: Toward a finer-grained methodological perspective. In Sanz, Cristina; Leow, Ronald P. (Hrsg.), Implicit and explicit conditions, processes and knowledge in SLA and bilinguism, 61-72. Washington, D.C.: Georgetown University Press.

Lightbown, Patsy M. 1998. The importance of timing in focus on form. In Doughty, Catherine; Williams, Jessica (Hrsg.), Focus on form in classroom second language acquisition, 177-196. Cambridge: Cambridge University Press.

Lightbown, Patsy M. 2000. Anniversary article: Classroom SLA research and second language teaching. Applied Linguistics 21(4). 431-462. https://doi.org/10.1093/applin/21.4.431

Master, Peter. 1994. The effect of systematic instruction on learning the English article system. In Odlin, Terence (Hrsg.), Perspectives on pedagogical grammar, 229-252. New York: Cambridge University Press.

Medina, Almitra. 2015. The variable effects of level of awareness and CALL versus nonCALL textual modification on adult L2 readers' input comprehension and learning. In Leow, Ronald P.; Cerezo, Luis; Baralt, Melissa (Hrsg.), A psycholinguistic approach to technology and language learning, 243-257. Berlin: De Gruyter Mouton.

Norris, John M.; Ortega, Lourdes. 2000. Effectiveness of L2 instruction: A research synthesis and quantitative meta-analysis. Language Learning 50(3). 417-528. https://doi.org/10.1111/0023-8333.00136

Posner, Michael I. 1992. Attention as a cognitive and neural system. Current Directions in Psychological Sciences 1(1). 11-14. https://doi.org/10.1111/1467-8721.ep10767759

Radwan, Adel Abu. 2005. The effectiveness of explicit attention to form in language learning. System 33(1). 69-87. https://doi.org/10.1016/j.system.2004.06.007

Reber, Arthur S. 1967. Implicit learning of artificial grammars. Journal of Verbal Learning and Verbal Behavior 61(6). 855-863.

Reber, Arthur S. 1969. Transfer of syntactic structure in synthetic languages. Journal of Experimental Psychology 81(1). 115-119. https://doi.org/10.1037/h0027454

Renou, Janet. 2001. An examination of the relationship between metalinguistic awareness and second language proficiency of adult learners of French. Language Awareness 10(4). 249-267. https://doi.org/10.1080/09658410108667038

Richards, Jack C.; Schmidt, Richard. 2002. Longman dictionary of language teaching and applied linguistics. Third edition. New York: Pearson education. 
Robinson, Peter. 1995. Attention, memory, and the "Noticing" Hypothesis. Language Learning 45(2). 283-331. https://doi.org/10.1111/j.1467-1770.1995.tb00441.x

Robinson, Peter. 1996. Learning simple and complex second language rules under implicit, incidental, rule-search, and instructed conditions. Studies in Second Language Acquisition 18(1). 27-67.

Robinson, Peter. 1997. Generalizability and automaticity of second language learning under implicit, incidental, enhanced, and instructed conditions. Studies in Second Language Acquisition 19(2). 223-247. https://doi.org/10.1017/S0272263197002052

Roehr, Karen. 2007. Metalinguistic knowledge and language ability in university-level L2 learners. Applied Linguistics 29(2). 173-199. https://doi.org/10.1093/applin/amm037

Rosa, Elena; Leow, Ronald P. 2004. Awareness, different learning conditions, and second language development. Applied Psycholinguistics 25(2). 269-292. https://doi.org/10.1017/S0142716404001134

Rosa, Elena; O’Neill, Michael D. 1999. Explicitness, intake, and the issue of awareness. Studies in Second Language Acquisition 21(4). 511-556.

Sachs, Rebecca; Suh, Bo-Ram. 2007. Textually enhanced recasts, learner awareness, and L2 outcomes in synchronous computer-mediated interaction. In Mackey, Alison (Hrsg.) Conversational interaction in second-language acquisition: A series of empirical studies, 197-227. Oxford: Oxford University Press.

Sachs, Rebecca; Hamrick, Phillip; McCormick, Timothy; Leow, Ronald P. 2020. Exploring the veridicality and reactivity of subjective measures of awareness: Is a "guess" really a guess? Studies in Second Language Acquisition 42(4). 919-932.

Schmidt, Richard W. 1990. The role of consciousness in second language learning. Applied Linguistics 11(2). 129-158. https://doi.org/10.1093/applin/11.2.129

Schmidt, Richard W. 1993. Awareness and second language acquisition. Annual Review of Applied Linguistics 13. 206-226.

Schmidt, Richard W. 1994. Implicit learning and the cognitive unconscious. In Ellis, Nick (Hrsg.), Implicit and explicit learning of languages, 165-209. London: Academic Press.

Schmidt, Richard W. 1995. Consciousness and foreign language learning: A tutorial on the role of attention and awareness in learning. In Schmidt, Richard W. (Hrsg.), Attention and awareness in foreign language learning, 1-63. Honolulu: University of Hawaii.

Schmidt, Richard W. 2001. Attention. In Robinson, Peter (Hrsg.), Cognition and second language instruction, 3-32. Cambridge: Cambridge University Press.

Schmidt, Richard W. 2010. Attention, awareness, and individual differences in language learning. In Chi, W. M.; Cin, K. N.; Istanto, J.; Nagami, M.; Sew, J. W.; Suthiwan, T.; Walker, I. (Hrsg.), Proceedings of ClaSIC 2010, Singapore, Dec 2-4, 721-737. Singapore: National University of Singapore, Centre for Language studies.

Scott, Virginia M. 1989. An empirical study of explicit and implicit teaching strategies in 
French. The Modern Language Journal 73(1). 14-22. https://doi.org/10.1111/j.15404781.1989.tb05303.x

Scott, Virginia M. 1990. Explicit and implicit grammar teaching: New empirical data. The French Review 63(5). 779-789.

Spada, Nina; Lightbown, Patsy M. 1999. Instruction, L1 influence and developmental readiness in second language acquisition. Modern Language Journal 83(2). 1-22. https://doi.org/10.1111/0026-7902.00002

Spada, Nina; Lightbown, Patsy M.; White, Joanna L. 2005. The importance of form/ meaning mappings in explicit form-focused instruction. In Housen, Alex; Pierrard, Michel (Hrsg.), Current issues in instructed second language learning, 199-234. Berlin: Mouton de Gruyter. https://doi.org/10.1515/9783110197372.2.199

Spada, Nina; Tomita, Yasuyo. 2010. Interactions between type of instruction and type of language feature: A meta-analysis. Language Learning 60(2). 263-308. https://doi.org/10.1111/j.1467-9922.2010.00562.x

Tomlin, Russell S.; Villa, Victor. 1994. Attention in cognitive science and second language acquisition. Studies in Second Language Acquisition 16(2). 183-203.

VanPatten, Bill. 1996. Input processing and grammar instruction in second language acquisition. Norwood: Ablex Publishing.

White, Lydia. 1991. Adverb placement in second language acquisition: Some positive and negative evidence in the classroom. Second Language Research 7(2). 133-161. https://doi.org/10.1177/026765839100700205

White, Joanna; Ranta, Leila. 2002. Examining the interface between metalinguistic task performance and oral production in a second language. Language Awareness 11(4). 259-290. https://doi.org/10.1080/09658410208667060

Williams, John N. 1999. Memory, attention, and inductive learning. Studies in Second Language Acquisition 21(1). 1-48.

Williams, Jessica; Evans, Jacqueline. 1998. What kind of focus and on which forms? In Doughty, Catherine; Williams, Jessica (Hrsg.), Focus on form in classroom second language acquisition, 139-155. Cambridge: Cambridge University Press.

Winitz, Harris 1996. Grammaticality judgment as a function of explicit and implicit instruction in Spanish. The Modern Language Journal 80(1). 32-46. https://doi.org/10.1111/j.1540-4781.1996.tb01135.x 


\section{Anhang 1}

\section{Pretest und verzögerter Posttest}

\section{Aufgabe 1:}

Lesen Sie sich bitte die Sätze genau durch. Was glauben Sie, welche Sätze sind sprachlich akzeptabel und grammatisch richtig? Kreuzen Sie an.

1. Den ganzen Abend wurde von uns diskutiert.

2. Das Päckchen wird der alten Dame vom Briefträger gegeben.

3. Bis jetzt wird wenig über die seltene Krankheit gewusst.

4. Die Suppe wurde vom Koch gekostet.

5. Nach dem Hurrikan wurde an Wasser gefehlt.

6. Ein Zentner wird vom Kater gewogen.

7. Die besten Plätze werden von den ersten Theaterbesuchern besetzt.

8. Von ihm wurde sein gesamter Mut gefasst, um ihr einen Heiratsantrag zu stellen.

9. Das Fleisch wurde vom Metzger gewogen.

10. Als zum Ausflug gegangen wurde, war die ganze Klasse gut gelaunt.

\section{Aufgabe 2:}

Lesen Sie sich bitte die Sätze genau durch. Kreuzen Sie nur die sprachlich akzeptablen und grammatisch richtigen Sätze an. Erklären Sie kurz, warum Sie glauben, dass genau diese Sätze sprachlich akzeptabel und grammatisch richtig sind, oder warum sie nicht sprachlich akzeptabel und nicht grammatisch richtig sind. Sie müssen ihre Erklärung nicht aufschreiben. Denken Sie laut nach und sprechen Sie dabei ins Mikrofon.

1. Von den Schülern wird in die Lehrbücher gesehen.

2. Als er spät von der Party nach Hause kam, wurde er von der Mutter durch die Haustür kommen gesehen.

3. Dem Vater wird vom Sohn geholfen.

4. Vom ihm wird Spanisch mit Leichtigkeit gekonnt.

5. Jeden Tag wird eine wilde Tierart ausgestorben, ohne dass vom Menschen etwas dagegen getan wird.

6. Die Studenten sind von ihren Professoren herzlich empfangen worden.

7. Dann wurden sich noch schnell die Zähne geputzt und es ging ab ins Bett.

8. Es wurde von dem Lehrer eine komplizierte Aufgabe bekommen.

9. Babys werden von ihren Müttern regelmäßig gewaschen.

10. Das Kind wurde vom Auto auf dem Zebrastreifen erfasst. 


\section{Aufgabe 3:}

a) Formulieren Sie die folgenden Sätze nach dem angegebenen Muster um:

1976 führte die Bundesrepublik die Gurtpflicht für Autofahrer ein.

$\rightarrow 1976$ wurde von der Bundesrepublik die Gurtpflicht für Autofahrer eingeführt. oder

$\rightarrow$ Es wurde 1976 von der Bundesrepublik die Gurtpflicht für Autofahrer eingeführt.

Passen Sie auf, nicht alle Sätze lassen sich transformieren!

b) Berichten Sie laut ins Mikrofon darüber, was Sie dazu gebracht hat, einige Sätze zu transformieren, andere aber nicht. Fangen Sie mit Satz 1 an und berichten Sie über jeden Satz einzeln.

\section{Macht Sicherheit sorglos?}

1. Seit 1985 bestraft man die Autofahrer ohne Gurt mit Bußgeld.

2. Gegner der Anschnallpflicht zitieren gerne Statistiken.

3. Die Zahl der tödlichen Unfälle von Autofahrern geht seit der Einführung der Gurtpflicht zurück.

4. Es starben aber mehr Radfahrer und Fußgänger bei Unfällen.

5. Die angeschnallten Autofahrer zählt man zu einer besonderen Risikogruppe.

6. Man weiß das aus verschiedenen Untersuchungen.

7. Menschen fühlen sich sicherer und werden immer risikobereiter.

8. Es gibt verschiedene gefährliche Studien zu diesem Thema.

9. Psychologen berichteten bereits 1976 davon. 


\section{Anhang 2}

\section{Unmittelbarer Posttest}

\section{Aufgabe 1:}

Lesen Sie sich bitte die Sätze genau durch. Was glauben Sie, welche Sätze sind sprachlich akzeptabel und grammatisch richtig? Kreuzen Sie an.

1. Das Mädchen wurde auf offener Straße vom Auto ergriffen.

2. Die besten Plätze wurden von den ersten Fußballfans reserviert.

3. Nach dem Sturm wurde es an Nahrung gemangelt.

4. Der Brief wurde der alten Dame vom Briefboten gegeben.

5. Von einem Meteoriten wurden alle Lebewesen vernichtet, die mehr als 20 Kilogramm gewogen wurden.

6. Die Vorspeise wurde von der Köchin probiert.

7. Von ihm wird schon lange in die Schule gemusst.

8. Es wird ein guter Facharzt für diese Krankheit von ihr gekannt.

9. Den ganzen Morgen wurde im Sprachunterricht geredet.

10. Das Gemüse wurde von der Verkäuferin gewogen.

\section{Aufgabe 2:}

Lesen Sie sich bitte die Sätze genau durch. Kreuzen Sie nur die sprachlich akzeptablen und grammatisch richtigen Sätze an. Erklären Sie kurz, warum Sie glauben, dass genau diese Sätze sprachlich akzeptabel und grammatisch richtig sind, oder warum, sie nicht sprachlich akzeptabel und nicht grammatisch richtig sind. Sie müssen ihre Erklärung nicht aufschreiben. Denken Sie laut nach und sprechen Sie dabei ins Mikrofon.

1. Als von der Klasse auf den Sportplatz gegangen wurde, waren alle gut gelaunt.

2. Das Kind wird von der Mutter getragen.

3. Kleinkinder werden von ihren Eltern regelmäßig umsorgt.

4. Dann wurden sich noch schnell die Hände gewaschen und es ging ab zum Mittagessen.

5. Von ihm wurde die Besinnung verloren, als er auf der Treppe fiel.

6. Als vom Unfall erfahren wurde, waren alle traurig.

7. Jeden Tag wird eine andere Tierart ausgestorben, ohne dass von den Menschen etwas Ernsthaftes dagegen unternommen wird.

8. Es wurde vom Mädchen ein Liebesbrief bekommen.

9. Die Schüler werden von ihren Lehrern herzlich begrüßt.

10. Es wurde heftig darüber diskutiert. 
Ivana Šarić Šokčević:

Die Beziehung zwischen der Bewusstheit und den Lehrmethoden beim Erlernen der (nicht) passivfähigen Verben im Deutschen als Fremdsprache

Aufgabe 3:

a) Formulieren Sie die folgenden Sätze nach dem angegebenen Muster um:

200 Millionen Jahre beherrschten die Saurier die Welt.

$\rightarrow$ Die Welt wurde 200 Millionen Jahre von den Sauriern beherrscht. oder

$\rightarrow 200$ Millionen Jahre wurde die Welt von den Sauriern beherrscht.

Wenn die Transformation nicht möglich ist, erklären Sie kurz warum.

b) Berichten Sie darüber, was Sie dazu gebracht hat, einige Sätze zu transformieren, andere aber nicht. Fangen Sie mit Satz 1 an und berichten Sie über jeden Satz einzeln.

\section{Warum starben die Saurier aus?}

1. Die Saurier zählen zu den größten Tieren, die die Menschheit kennt.

2. Vor 65 Millionen Jahren verschwanden sie dann plötzlich.

3. Dafür gibt es zahllose Erklärungen.

4. Vor 65 Millionen Jahren traf ein riesiger Meteorit die Erde.

5. Dieser Meteorit vernichtete fast alle Lebewesen, die mehr als 20 Kilogramm wogen.

6. Die Erde erholte sich davon erst nach Millionen von Jahren.

7. Heute bedroht der Mensch mit seinem Verhalten viele Tierarten.

8. Jeden Tag stirbt eine Tierart auf der Erde aus.

9. Viele Politiker und Organisationen versuchen, eine Lösung zu finden. 


\section{Anhang 3}

\section{Transkribierte Veranschaulichungsbeispiele zur Kodifizierung der Bewusstheitsebenen}

\begin{tabular}{|c|c|c|}
\hline & Transkribiertes Beispiel & Bewusstheitsebene \\
\hline 1 & $\begin{array}{l}\text { (Liest den Satz)...ma joj...taj erfahren..ne...ne može...a kako objasni- } \\
\text { ti...ok } \\
\text { (Unmittelbarer Posttest, implizite Lehrmethode, Gymnasium) }\end{array}$ & + Noticing \\
\hline 2 & $\begin{array}{l}\text { (Liest den Satz)...vor } 65 \text { mil jahren verschwanden sie...macht keinen } \\
\text { sinn...kann man nicht transformieren...nein...wie heißen diese ver- } \\
\text { ben...nein...ma ovo ne može nikako zbog verschwinden...tog značenja } \\
\text { (Unmittelbarer Posttest, explizite Lehrmethode, Gymnasium) }\end{array}$ & + Noticing \\
\hline 3 & $\begin{array}{l}\text { (Liest den Satz)...ma...nein...das geht nicht...es wurde gegangen...na što } \\
\text { bi to...ma na hrvatskome da...ali na njemačkome...ne može nikako zbog } \\
\text { gehen } \\
\text { (Unmittelbarer Posttest, explizite Lehrmethode, Fakultät) }\end{array}$ & + Noticing \\
\hline 4 & $\begin{array}{l}\text { (Liest den Satz)...uh....ovo iskreno nemam pojma što znači jer to uopće } \\
\text { ispravno...mangelt meni to... ne zvuči lijepo (liest den Satz) joj ne znam } \\
\text { to meni ružno zvuči...mislim da ne može zbog tog mangeln...to znače- } \\
\text { nje...kao nedostaje...nema nečega } \\
\text { (unmittelbar, implizit, faks) } \\
\text { (Unmittelbarer Posttest, implizite Lehrmethode, Gymnasium) }\end{array}$ & + Noticing \\
\hline 5 & $\begin{array}{l}\text { (Liest den Satz)...kannt...gekannt...mislim da ne...ružno mi zvuči...to je } \\
\text { kennen...jako mi ružno zvuči gekannt...gekennt werden...možda kennen } \\
\text { werden ostaje u infinitivu...werden ili wird ružno mi zvuči...ma to mi po } \\
\text { smislu nema smisla....kada prevedem..znan je...poznat je...da....ali ne mo- } \\
\text { že to nikako } \\
\text { (Unmittelbarer Posttest, implizite Lehrmethode, Gymnasium) }\end{array}$ & + Noticing \\
\hline 6 & $\begin{array}{l}\text { (Liest den Satz)...dieser satz macht keinen ...ich verstehe den satz aber } \\
\text { ich glaube nicht dass er akzeptabel ist...der LKW wiegt nicht die } 3 \text { ton- } \\
\text { nen...ma ne moram na njemačkome...kako bi to uopće transformi- } \\
\text { rao...ma ne može se to nikako...kako taj glagol prebacit... ma ne...ne } \\
\text { može nikako } \\
\text { (Verzögerter Posttest, implizite Lehrmethode, Fakultät) }\end{array}$ & +Noticing \\
\hline 7 & $\begin{array}{l}\text { (Liest den Satz)...ah...er kann er konnte...gekonnt...hmhm...es klingt } \\
\text { nicht richtig....nein dieses wird gekonnt...nein geht nicht } \\
\text { (Verzögerter, explizite Lehrmethode, Fakultät) }\end{array}$ & + Noticing \\
\hline 8 & $\begin{array}{l}\text { (Liest den Satz)...es ist nicht moeglich...sie wurden geworden...nein } \\
\text { (Verzögerter, implizite Lehrmethode, Fakultät) }\end{array}$ & + Noticing \\
\hline
\end{tabular}


Ivana Šarić Šokčević:

Die Beziehung zwischen der Bewusstheit und den Lehrmethoden beim Erlernen der (nicht) passivfähigen Verben im Deutschen als Fremdsprache

\begin{tabular}{|c|c|c|}
\hline 9 & $\begin{array}{l}\text { (Liest den Satz)...uf tututu...gegangen wurde...ružno izgle- } \\
\text { da...ne....wurden...nego bi možda išlo gegangen pošto gehen ide sa ist als } \\
\text { von der klasse ins schwimmbad gegeangen waren...da...war da...to je } \\
\text { glagol kretanja on ide sa ist...a ide sa ist zato što...to je glagol kreta- } \\
\text { nja...ma da... mislim da to nije dobro mislim...glagoli kretanja ne mogu } \\
\text { tvoriti pasiv...ima neka iznimka...ali to nije ova...ne...ovo ne može } \\
\text { (Unmittelbarer Posttest, explizite Lehrmethode, Fakultät) }\end{array}$ & + Understanding \\
\hline 10 & $\begin{array}{l}\text { (Liest den Satz)...(liest den Satz)...mangeln an...es...es mangelt an...to je } \\
\text { ono unpersönlich sa es pa se zato ne može transformirati u pasiv } \\
\text { (Unmittelbarer Posttest, explizite Lehrmethode, Gymnasium) }\end{array}$ & + Understanding \\
\hline 11 & $\begin{array}{l}\text { (Liest den Satz)...ne može jer imamo kilograme koliko su težili...ne mo- } \\
\text { že zbog glagola wiegen....koji označava ....koji u ovom slučaju označava } \\
\text { točan iznos kilograma } \\
\text { (Unmittelbarer Posttest, explizite Lehrmethode, Fakultät) }\end{array}$ & + Understanding \\
\hline 12 & $\begin{array}{l}\text { (Liest den Satz)...sich erholen...ne može jer je reflexiv...sich erholen...ist } \\
\text { ein reflexives verb....und kann deswegen kein pasiv bilden } \\
\text { (Unmittelbarer Posttest, explizite Lehrmethode, Gymnasium) }\end{array}$ & + Understanding \\
\hline 13 & $\begin{array}{l}\text { (Liest den Satz)...hmmm...kada se ide na izlet...ne može gegangen jer je } \\
\text { gehen...glagol kretanja...koji ne može u pasiv } \\
\text { (Verzögerter Posttest, explizite Lehrmethode, Fakultät) }\end{array}$ & + Understanding \\
\hline 14 & $\begin{array}{l}\text { (Liest den Satz)... (liest den Satz) ne može...ali ne znam zašto....kako da } \\
\text { kažem to sa wissen...ma taj glagol...wissen...zna se...znalo } \\
\text { se...hmmm..na hrvatskome bi moglo...ali ne na njemačkome ne...ne ku- } \\
\text { žim zašto...ali...ne...kliknut ću da ne može zbog wissen ...znano se...ne } \\
\text { može...to je kao nešto kognitivno...netko je nešto znao...nije neka rad- } \\
\text { nja...kao netko je nekoga udario ili...ili...tako...nekako } \\
\text { (Verzögerter Posttest, implizite Lehrmethode, Gymnasium) }\end{array}$ & + Understanding \\
\hline
\end{tabular}




\section{Anhang 4}

Treatmentablauf (Verlauf der Treatmentphase) für die explizite Lehrmethode und

Treatmen-tablauf für die implizite Lehrmethode

Treatmentablauf - Verlauf der Treatmentphase (explizite Lehrmethode)

\section{EINLEITUNG}

\begin{tabular}{|c|c|c|c|c|}
\hline $\begin{array}{l}\text { Phasen der } \\
\text { Stoffbearbei- } \\
\text { tung/Zeit }\end{array}$ & Lernziele & $\begin{array}{l}\text { Aktivitäten der Unter- } \\
\text { suchungsleiterin (UL) }\end{array}$ & $\begin{array}{l}\text { Aktivitäten der Untersuch- } \\
\text { ungsteilnehmer (UT) }\end{array}$ & $\begin{array}{l}\text { a) Arbeits-formen } \\
\text { b) Sozialformen, } \\
\text { Medien und Mate- } \\
\text { rialien }\end{array}$ \\
\hline \multirow[t]{2}{*}{$\begin{array}{l}\text { Einstieg/ } \\
\text { Vorentlastung } \\
5\end{array}$} & \multirow[t]{2}{*}{$\begin{array}{l}\text { Die Untersuchungsteilne- } \\
\text { hmer (UT) sollen richtige } \\
\text { Sätze im Vorgangspassiv zu } \\
\text { den entsprechenden Bildern } \\
\text { formulieren. }\end{array}$} & $\begin{array}{l}\text { 1.1.: Begrüßung/ Wiederho- } \\
\text { lung der Bildung des Vor- } \\
\text { gangspassivs - das Vorwis- } \\
\text { sen aktivieren } \\
\text { Die Untersuchungsleiterin } \\
\text { (UL) begrüßt die UT. Die } \\
\text { UL zeigt einige Bilder und } \\
\text { stellt den UT Fragen. Die } \\
\text { UL kontrolliert die Antwor- } \\
\text { ten. }\end{array}$ & $\begin{array}{l}\text { Die UT schauen sich die Bilder } \\
\text { auf der PowerPoint Präsentati- } \\
\text { on an und beschreiben die Bil- } \\
\text { der und/oder antworten auf die } \\
\text { Fragen der UL. }\end{array}$ & $\begin{array}{l}\text { a) Fragen und An- } \\
\text { tworten } \\
\text { b) Unterrich- } \\
\text { tsgespräch, } \\
\text { Computer }\end{array}$ \\
\hline & & $\begin{array}{l}1.2 .: \text { Wiederholung der Bil- } \\
\text { dung des Vorgangspassivs } \\
\text { das Vorwissen aktivieren } \\
\text { Die UL stellt den UT Fragen } \\
\text { bezüglich der Bildung und } \\
\text { Semantik des Vorgangspas- } \\
\text { siv des regelmäßigen Verbs } \\
\text { schießen. Die UL kontrolli- } \\
\text { ert die Antworten. }\end{array}$ & $\begin{array}{l}\text { Die UT bilden die verschiede- } \\
\text { nen Zeitformen im Vorgangs- } \\
\text { passiv. }\end{array}$ & $\begin{array}{l}\text { a) Fragen und An- } \\
\text { tworten, mündliches } \\
\text { Üben } \\
\text { b) Unterrich- } \\
\text { tsgespräch, Compu- } \\
\text { ter }\end{array}$ \\
\hline
\end{tabular}




\section{Ivana Šarić Šokčević: \\ Die Beziehung zwischen der Bewusstheit und den Lehrmethoden beim Er- lernen der (nicht) passivfähigen Verben im Deutschen als Fremdsprache}

\section{HAUPTTEIL}

\begin{tabular}{|c|c|c|c|c|}
\hline $\begin{array}{l}\text { Phasen der } \\
\text { Stoffbearbei- } \\
\text { tung/Zeit }\end{array}$ & Lernziele & Aktivitäten der Untersuchungsleiterin & $\begin{array}{l}\text { Aktivitäten der } \\
\text { Untersuchungs- } \\
\text { teilnehmer }\end{array}$ & $\begin{array}{l}\text { a) Arbeits- } \\
\text { formen } \\
\text { b) Sozialfor- } \\
\text { men, Medien } \\
\text { und Materiali- } \\
\text { en }\end{array}$ \\
\hline \multirow[t]{2}{*}{$\begin{array}{l}\text { Darbietung } \\
\text { des neuen } \\
\text { Materials/ } \\
\text { Einübung } \\
40^{6}\end{array}$} & $\begin{array}{l}\text { Die UT sollen mithilfe der vor- } \\
\text { gestellten Einschränkungen } \\
\text { diejenigen Sätze in das Vor- } \\
\text { gangspassiv umformulieren, die } \\
\text { transformiert werden können. } \\
\text { Falls ein Satz nicht ins Vor- } \\
\text { gangspassiv umformuliert wer- } \\
\text { den kann, sollen die UT mithilfe } \\
\text { der vorgestellten Einschränkun- } \\
\text { gen erklären, warum eine Trans- } \\
\text { formation ausgeschlossen ist. }\end{array}$ & $\begin{array}{l}\text { 2.1.: Sätze umformulieren } \\
\text { Die UL erklärt den UT die einzelnen Ein- } \\
\text { schränkungen bezüglich der Bildung des } \\
\text { Vorgangspassivs. Die UL fängt mit der ers- } \\
\text { ten Einschränkung an. Die UL löst jeweils } \\
\text { das erste Beispiel der jeweiligen Aufgabe. } \\
\text { Es gibt insgesamt sechs Aufgaben, die sich } \\
\text { auf die einzelnen Einschränkungen bezi- } \\
\text { ehen. } \\
\text { (Aufgabe 1: Bei welchen Verben ist ein } \\
\text { "Täter"/ein Agens denkbar? Unterstreichen } \\
\text { Sie die passivfähigen Verben. Aufgabe 2: } \\
\text { Formen Sie die folgenden Sätze mit Akkusa- } \\
\text { tiv- wenn möglich-in das Vorgangspassiv } \\
\text { um und erklären Sie - im negativen Falle-, } \\
\text { warum das nicht möglich ist. Aufgabe 3: }\end{array}$ & $\begin{array}{l}\text { Die UT lösen Au- } \\
\text { fgabe für Aufga- } \\
\text { be und stellen, } \\
\text { wenn notwendig, } \\
\text { Fragen zu den e- } \\
\text { inzelnen Ein- } \\
\text { schränkungen } \\
\text { und transformier- } \\
\text { ten Sätzen. }\end{array}$ & $\begin{array}{l}\text { a) Lesen, } \\
\text { Schreiben } \\
\text { b) Einzelarbeit, } \\
\text { Arbeitsblatt }\end{array}$ \\
\hline & & $\begin{array}{l}\text { Formen Sie die folgenden Sätze mit Akkusa- } \\
\text { tiv - wenn möglich - in das Vorgangspassiv } \\
\text { um und erklären Sie - im negativen Falle -, } \\
\text { warum das nicht möglich ist. Aufgabe 4: Ist } \\
\text { es möglich die folgenden Sätze mit Akkusativ } \\
\text { in das Vorgangspassiv umzuformen? Au- } \\
\text { fgabe 5: Formen Sie die folgenden Sätze mit } \\
\text { Akkusativ - wenn möglich - in das Vor- } \\
\text { gangspassiv um und erklären Sie - im nega- } \\
\text { tiven Falle -, warum das nicht möglich ist. } \\
\text { Aufgabe 6: Formen Sie die folgenden Sätze } \\
\text { mit Akkusativ - wenn möglich - in das Vor- } \\
\text { gangspassiv um und erklären Sie - im nega- } \\
\text { tiven Falle -, warum das nicht möglich ist.) } \\
\text { Die UL überprüft mit den UT die Lösungen } \\
\text { nach jeder einzelnen Aufgabe und erklärt } \\
\text { vor allem die Beispiele, die kein Vorgangs- } \\
\text { passiv bilden können. }\end{array}$ & & \\
\hline $\begin{array}{l}\text { Festigung } \\
20^{\circ}\end{array}$ & $\begin{array}{l}\text { Die UT sollen mithilfe der vor- } \\
\text { gestellten Einschränkungen die } \\
\text { Sätze in das Vorgangspassiv um- } \\
\text { formulieren, die transformiert } \\
\text { werden können. Falls ein Satz }\end{array}$ & $\begin{array}{l}\text { 2.2: Sätze umformulieren } \\
\text { Die UL erklärt den UT Aufgabe } 7 \text { (Formen } \\
\text { Sie die folgenden Sätze mit Akkusativ - } \\
\text { wenn möglich - in das Vorgangspassiv um } \\
\text { und erklären Sie - im negativen Falle -, }\end{array}$ & $\begin{array}{l}\text { Die UT lösen Au- } \\
\text { fgabe } 7 \text {. Sie for- } \\
\text { mulieren die } \\
\text { entsprechenden } \\
\text { Sätze, wenn }\end{array}$ & $\begin{array}{l}\text { a) Lesen, } \\
\text { Schreiben } \\
\text { b) Partnerarbeit, } \\
\text { Arbeitsblatt }\end{array}$ \\
\hline & $\begin{array}{l}\text { nicht ins Vorgangspassiv trans- } \\
\text { formiert werden kann, sollen die } \\
\text { UT mithilfe der vorgestellten E- } \\
\text { inschränkungen erklären, warum } \\
\text { eine Transformation a- } \\
\text { usgeschlossen ist. }\end{array}$ & $\begin{array}{l}\text { warum das nicht möglich ist.) auf dem Arbe- } \\
\text { itsblatt. Die UL überprüft mit den UT die } \\
\text { Lösungen und gibt gegebenenfalls noch wei- } \\
\text { tere Beispiele, um die zugrunde liegende E- } \\
\text { inschränkung so gut wie möglich zu er- } \\
\text { klären. }\end{array}$ & $\begin{array}{l}\text { möglich, in das } \\
\text { Vorgangspassiv } \\
\text { um. Die Sätze } \\
\text { sind nicht in ei- } \\
\text { nen Kontext ein- } \\
\text { gebettet. }\end{array}$ & \\
\hline
\end{tabular}




\section{SCHLUSSTEIL}

\begin{tabular}{|c|c|c|c|c|}
\hline $\begin{array}{l}\text { Phasen der } \\
\text { Stoffbearbei- } \\
\text { tung/Zeit }\end{array}$ & Ziele & $\begin{array}{l}\text { Aktivitäten der Untersuchungslei- } \\
\text { terin }\end{array}$ & $\begin{array}{l}\text { Aktivitäten der Unter- } \\
\text { suchungsteilnehmer }\end{array}$ & $\begin{array}{l}\text { a) Arbeits- } \\
\text { formen } \\
\text { b) Sozialfor- } \\
\text { men, Medien } \\
\text { und Mate- } \\
\text { rialien }\end{array}$ \\
\hline $\begin{array}{l}\text { Anwendung/Transfer } \\
23^{\circ}\end{array}$ & $\begin{array}{l}\text { Die UT sollen mithilfe } \\
\text { der vorgestellten Ein- } \\
\text { schränkungen erken- } \\
\text { nen, welche Sätze im } \\
\text { Text transformiert } \\
\text { werden können und } \\
\text { welche nicht. Falls ein } \\
\text { Satz nicht ins Vor- } \\
\text { gangspassiv transfor- } \\
\text { miert werden kann, sol- } \\
\text { len die UT mithilfe der } \\
\text { vorgestellten Ein- } \\
\text { schränkungen erklären, } \\
\text { warum eine Transfor- } \\
\text { mation ausgeschlossen } \\
\text { ist. }\end{array}$ & $\begin{array}{l}\text { 3.1: Einen Text transformieren } \\
\text { Die UL erklärt den UT Aufgabe 8. a) } \\
\text { (Formen Sie folgende Sätze aus dem } \\
\text { Aktiv in das Vorgangspassiv um und } \\
\text { wenn die Bildung des Passivs nicht } \\
\text { möglich ist, erklären Sie kurz } \\
\text { warum.) auf dem Arbeitsblatt. Die } \\
\text { UL überprüft mit den UT die } \\
\text { Lösungen und gibt gegebenenfalls } \\
\text { noch weitere Beispiele, um die zu- } \\
\text { grunde liegende Einschränkung so } \\
\text { gut wie möglich zu erklären. } \\
\text { *Falls noch etwas Zeit übrig ist, } \\
\text { entscheidet die UL sich für die Zu- } \\
\text { satzaufgabe 8.b) (Formen Sie fol- } \\
\text { gende Sätze aus dem Aktiv in das } \\
\text { Vorgangspassiv um und wenn die }\end{array}$ & $\begin{array}{l}\text { Die UT lösen Aufgabe } 8 . \\
\text { a). Sie transformieren die } \\
\text { entsprechenden Sätze aus } \\
\text { dem Text ,Ist schwarzfa- } \\
\text { hren politisch korrekt?“, } \\
\text { wenn möglich, in das } \\
\text { Vorgangspassiv um. Die } \\
\text { UT geben eine Erklärung } \\
\text { in Form der entsprechen- } \\
\text { den Einschränkung für } \\
\text { die Sätze, die man nicht } \\
\text { ins Vorgangspassiv trans- } \\
\text { formieren kann. Die UT } \\
\text { arbeiten an einem Text } \\
\text { und nicht an isolierten, } \\
\text { dekontextualisierten Sät- } \\
\text { zen. }\end{array}$ & $\begin{array}{l}\text { a) Lesen, } \\
\text { Schreiben } \\
\text { b) Partnerarbe- } \\
\text { it, Arbei- } \\
\text { tsblatt }\end{array}$ \\
\hline & & $\begin{array}{l}\text { Bildung des Passivs nicht möglich } \\
\text { ist, erklären Sie kurz warum). }\end{array}$ & & \\
\hline $2^{6}$ & $\begin{array}{l}\text { Die UT sollen die Ein- } \\
\text { schränkungen au- } \\
\text { fzählen oder nennen, } \\
\text { die sie in der Stunde } \\
\text { gelernt haben. }\end{array}$ & $\begin{array}{l}\text { 3.2: Zusammenfassung } \\
\text { Die UL fragt die UT, was sie in der } \\
\text { Stunde gelernt haben. Die UL kon- } \\
\text { trolliert die Antworten. Die UL be- } \\
\text { dankt sich und verabschiedet sich. }\end{array}$ & $\begin{array}{l}\text { Die UT sollen die Ein- } \\
\text { schränkungen aufzählen, } \\
\text { die sie in der Stunde ge- } \\
\text { lernt haben. }\end{array}$ & $\begin{array}{l}\text { a) Fragen und } \\
\text { Antworten } \\
\text { b) Plenum }\end{array}$ \\
\hline
\end{tabular}


Ivana Šarić Šokčević:

Die Beziehung zwischen der Bewusstheit und den Lehrmethoden beim Erlernen der (nicht) passivfähigen Verben im Deutschen als Fremdsprache

\section{Treatmentablauf - Verlauf der Treatmentphase (implizite Lehrmethode)}

\section{EINLEITUNG}

\begin{tabular}{|c|c|c|c|c|c|}
\hline $\begin{array}{l}\text { Phasen der } \\
\text { Stoffbearbe } \\
\text { itung/Zeit }\end{array}$ & Lernziele & $\begin{array}{l}\text { Aktivitäten der Untersuchungsleite- } \\
\text { rin (UL) }\end{array}$ & $\begin{array}{l}\text { Aktivitäten der } \\
\text { Untersuchungste- } \\
\text { ilnehmer (UT) }\end{array}$ & $\begin{array}{l}\text { a) Arbeits-formen } \\
\text { b) Sozialformen, } \\
\text { Medien und Mate- } \\
\text { rialien Arbeits- } \\
\text { formen }\end{array}$ & $\begin{array}{l}\text { Didaktischer } \\
\text { Kommentar }\end{array}$ \\
\hline $\begin{array}{l}\text { Einstieg/ } \\
\text { Vorentlas- } \\
\text { tung } \\
8^{6}\end{array}$ & $\begin{array}{l}\text { Untersuch- } \\
\text { ungs- } \\
\text { teilnehmer } \\
\text { (UT) sollen } \\
\text { das vorge- } \\
\text { stellte Bild } \\
\text { beschreiben. }\end{array}$ & $\begin{array}{l}1 . \text { Begrüßung/ Wiederholung des } \\
\text { Wortschatzes und der Bildung des } \\
\text { Vorgangspassivs-das Vorwissen akti- } \\
\text { vieren } \\
\text { Die Untersuchungsleiterin (UL) be- } \\
\text { grüßt die UT. Die UL zeigt den UT } \\
\text { per PowerPoint ein Bild, das eine Fa- } \\
\text { milie zeigt (siehe Anhang). Die UL } \\
\text { erteilt den UT die Aufgabe, das Bild } \\
\text { mündlich zu beschreiben (Was mac- } \\
\text { hen die Familienmitglieder auf dem } \\
\text { Bild? Wie fuihlen sie sich wahrschei- } \\
\text { nlich? Wer ist der Großvater, } \\
\text { Großmutter usw.? } \\
\text { Die UL wiederholt jedes Mal, wenn } \\
\text { die UT einen richtigen Satz zum Bild } \\
\text { formulieren, und transformiert diesen } \\
\text { ins Vorgangspassiv um, wenn möglich }\end{array}$ & $\begin{array}{l}\text { Die UT beschrei- } \\
\text { ben das per } \\
\text { PowerPoint gezei- } \\
\text { gte Bild und an- } \\
\text { tworten gegebe- } \\
\text { nenfalls auf Fra- } \\
\text { gen }\end{array}$ & $\begin{array}{l}\text { a) Fragen und An- } \\
\text { tworten, mündlic- } \\
\text { hes Üben } \\
\text { b) Unterrichts- } \\
\text { gespräch, } \\
\text { Bild, Computer }\end{array}$ & $\begin{array}{l}\text { Hier ist es wichtig, } \\
\text { den UT implizit da- } \\
\text { rauf aufmerksam zu } \\
\text { machen, dass einige } \\
\text { Sätze ins Vorgangs- } \\
\text { passiv umformuliert } \\
\text { werden können, an- } \\
\text { dere nicht. }\end{array}$ \\
\hline & & $\begin{array}{l}\text { (z.B. Sie machen ein Selfie. } \rightarrow \text { Es wird } \\
\text { ein Selfie gemacht.). Die UL kontrol- } \\
\text { liert die Antworten. }\end{array}$ & & & \\
\hline
\end{tabular}




\section{HAUPTTEIL}

\begin{tabular}{|c|c|c|c|c|c|}
\hline $\begin{array}{l}\text { Phasen der } \\
\text { Stoffbearbei- } \\
\text { tung/Zeit }\end{array}$ & Lernziele & $\begin{array}{l}\text { Aktivitäten der Untersuchungs- } \\
\text { leiterin }\end{array}$ & $\begin{array}{l}\text { Aktivitäten der Unter- } \\
\text { suchungsteilnehmer }\end{array}$ & $\begin{array}{l}\text { a) Arbeits- } \\
\text { formen } \\
\text { b) Sozialfor- } \\
\text { men, Medien } \\
\text { und Materia- } \\
\text { lien }\end{array}$ & $\begin{array}{l}\text { Didaktischer } \\
\text { Kommentar }\end{array}$ \\
\hline $\begin{array}{l}\text { Darbietung } \\
\text { des } \\
\text { neuen Mate- } \\
\text { rials } \\
7^{\circ}\end{array}$ & $\begin{array}{l}\text { Die UT sollen } \\
\text { während des } \\
\text { Lesens das, } \\
\text { was Marko } \\
\text { macht, unter- } \\
\text { streichen. }\end{array}$ & $\begin{array}{l}\text { 2.1.: selektives Lesen } \\
\text { Die UL erklärt Aufgabe 1. a) } \\
\text { (Unterstreichen Sie im Text, was } \\
\text { Marko alles macht.). } \\
\text { Die UL kontrolliert die Aufgabe. }\end{array}$ & $\begin{array}{l}\text { Die UT lesen den Text und } \\
\text { unterstreichen die } \\
\text { entsprechenden Informatio- } \\
\text { nen. }\end{array}$ & $\begin{array}{l}\text { a) Lesen, } \\
\text { Schreiben } \\
\text { b) Einzelarbe- } \\
\text { it, Arbei- } \\
\text { tsblatt, Com- } \\
\text { puter }\end{array}$ & \\
\hline $8^{6}$ & $\begin{array}{l}\text { Die UT sollen } \\
\text { die Informati- } \\
\text { onen aus dem } \\
\text { Text in die } \\
\text { richtige Spalte } \\
\text { einordnen.. }\end{array}$ & $\begin{array}{l}\text { 2.2.: Sortieren } \\
\text { Die UL erklärt den UT Aufgabe } \\
\text { 1.b) (Tragen Sie weitere Informa- } \\
\text { tionen iiber Marko unter der pas- } \\
\text { senden Überschrift in die Tabelle } \\
\text { ein.) auf dem Arbeitsblatt. Die } \\
\text { UL fordert die UT auf, noch min- } \\
\text { destens zwei eigene Beispiele da- } \\
\text { zuzuschreiben. Die UL überprüft } \\
\text { mit den UT die Iösungen. }\end{array}$ & $\begin{array}{l}\text { Die UT sortieren die Infor- } \\
\text { mationen und schreiben sie } \\
\text { in die richtige Spalte. Sie } \\
\text { fügen auch die Informatio- } \\
\text { nen aus Aufgabe 1.a) hinzu. }\end{array}$ & $\begin{array}{l}\text { a) Lesen, } \\
\text { Schreiben } \\
\text { b) Einzelarbe- } \\
\text { it, Arbei- } \\
\text { tsblatt, Com- } \\
\text { puter }\end{array}$ & $\begin{array}{l}\text { Die UL gibt kei- } \\
\text { ne expliziten Er- } \\
\text { klärungen } \\
\text { bezüglich der } \\
\text { Antworten, selbst } \\
\text { wenn sie von den } \\
\text { UT nach diesen } \\
\text { gefragt wird. }\end{array}$ \\
\hline $10^{\circ}$ & $\begin{array}{l}\text { Die UT sollen } \\
\text { einen kurzen } \\
\text { Text nach dem } \\
\text { angegeben } \\
\text { Muster schrei- } \\
\text { ben. }\end{array}$ & $\begin{array}{l}\text { 2.3.: Gelenktes Produzieren } \\
\text { Die UL erklärt den UT Aufgabe } \\
\text { 1. c) (Schreiben Sie eine kurze } \\
\text { Geschichte nach demselben Mus- } \\
\text { ter über sich. Vergleichen Sie } \\
\text { sich dabei mit einer Person, mit } \\
\text { der Sie sehr viel gemeinsam ha- } \\
\text { ben. Beziehen Sie sich dabei auf } \\
\text { die folgenden Bereiche: das Aus- } \\
\text { sehen, die Hobbys, den Charak- } \\
\text { ter:) Die UL bietet Hilfestellung } \\
\text { bei der Lösung der Aufgabe und } \\
\text { kontrolliert am Ende das entstan- } \\
\text { dene Produkt. }\end{array}$ & $\begin{array}{l}\text { Die UT schreiben eine kurze } \\
\text { Geschichte nach demselben } \\
\text { Muster wie in Aufgabe 1. a), } \\
\text { aber über sich. Die UT ver- } \\
\text { gleichen sich dabei mit einer } \\
\text { Person, mit der sie sehr viel } \\
\text { gemeinsam haben, wobei sie } \\
\text { sich dabei auf die folgenden } \\
\text { Bereiche beziehen: das Aus- } \\
\text { sehen, die Hobbys, den Cha- } \\
\text { rakter. }\end{array}$ & $\begin{array}{l}\text { a) Schreiben, } \\
\text { Zuhören } \\
\text { b) Einzelarbe- } \\
\text { it, Arbei- } \\
\text { tsblatt, Com- } \\
\text { puter }\end{array}$ & $\begin{array}{l}\text { Die UL gibt nur } \\
\text { implizites Feed- } \\
\text { back und keine } \\
\text { expliziten Er- } \\
\text { klärungen } \\
\text { bezüglich der } \\
\text { Bildung der Sät- } \\
\text { ze, selbst wenn } \\
\text { die UT nach } \\
\text { konkreten Re- } \\
\text { geln fragen sol- } \\
\text { lten. }\end{array}$ \\
\hline $\begin{array}{l}\text { Einübung } \\
10^{\circ}\end{array}$ & $\begin{array}{l}\text { Die UT sollen } \\
\text { richtige Sätze } \\
\text { mit dem vor- } \\
\text { gegebenen } \\
\text { Wortmaterial } \\
\text { bilden. }\end{array}$ & $\begin{array}{l}\text { 2.4.: Sätze (mündlich) bilden } \\
\text { Die UL erklärt die nächste Au- } \\
\text { fgabe auf dem Arbeitsblatt (Au- } \\
\text { fgabe 2: Vor und während der } \\
\text { Geburtstagsparty. Was wird vor } \\
\text { der Party gemacht, und } \\
\text { was wird während der Party ge- }\end{array}$ & $\begin{array}{l}\text { Die UT bilden Sätze } \\
\text { darüber, was vor und } \\
\text { während einer Geburts- } \\
\text { tagsparty gemacht wird, was } \\
\text { alles vorbereitet wird bzw. } \\
\text { was alles passiert, wobei sie } \\
\text { das angegebene Wortmateri- }\end{array}$ & $\begin{array}{l}\text { a) Lesen, } \\
\text { Schreiben } \\
\text { b) Partne- } \\
\text { rarbeit, Arbei- } \\
\text { tsblatt, Com- } \\
\text { puter }\end{array}$ & \\
\hline
\end{tabular}




\section{Ivana Šarić Šokčević: \\ Die Beziehung zwischen der Bewusstheit und den Lehrmethoden beim Er- lernen der (nicht) passivfähigen Verben im Deutschen als Fremdsprache}

\begin{tabular}{|c|c|c|c|c|c|}
\hline & $\begin{array}{l}\text { Die UT sollen } \\
\text { schriftlich } \\
\text { richtige Sätze } \\
\text { mit dem vor- } \\
\text { gegebenen } \\
\text { Wortmaterial } \\
\text { bilden. }\end{array}$ & $\begin{array}{l}\text { macht? Was wird alles vorberei- } \\
\text { tet? Was passiert alles? } \\
\text { Benutzen Sie auch das folgende } \\
\text { Wortmaterial: putzen - einkaufen } \\
\text { - Musik vorbereiten - sich freuen } \\
\text { - Staub saugen - singen - sich } \\
\text { unterhalten - begeistert sein - } \\
\text { tanzen - eine Torte haben - essen } \\
\text { - trinken - Spaß haben - sich be- } \\
\text { dienen - zu spät kommen - in die } \\
\text { Küche miissen - etwas zerbricht). } \\
\text { Die UL gibt ein Beispiel. Die UL } \\
\text { überprüft mit den UT die Lösun- } \\
\text { gen. Dazu ruft die UL einige UT } \\
\text { auf, um ihre Lösungen vorzulesen }\end{array}$ & al benutzen & & \\
\hline $10^{\circ}$ & $\begin{array}{l}\text { Die UT sollen } \\
\text { Fragen zum } \\
\text { Text be- } \\
\text { antworten }\end{array}$ & $\begin{array}{l}\text { 2.5.: Sätze (schriftlich) bilden } \\
\text { Die UL erklärt Aufgabe } 3 \\
\text { (Beschreiben Sie das Bild. Was } \\
\text { wird alles am Bahnhof in Fran- } \\
\text { kfurt am Main gemacht? Was } \\
\text { passiert?). } \\
\text { auf dem Arbeitsblatt. } \\
\text { Die UL gibt ein Beispiel. }\end{array}$ & $\begin{array}{l}\text { Die UT beschreiben schrif- } \\
\text { tlich mithilfe des vorgegebe- } \\
\text { nen Wortmaterials das Bild } \\
\text { „Am Bahnhof in Frankfurt } \\
\text { am Main“ in Aufgabe } 3 .\end{array}$ & $\begin{array}{l}\text { a) Lesen, } \\
\text { mündliches } \\
\text { Üben } \\
\text { b) Partne- } \\
\text { rarbeit, Arbei- } \\
\text { tsblatt, Com- } \\
\text { puter }\end{array}$ & $\begin{array}{l}\text { Visuelles } \\
\text { Input enhan- } \\
\text { cement durch } \\
\text { Farben }\end{array}$ \\
\hline & & $\begin{array}{l}\text { Die UL überprüft mit den UT die } \\
\text { Lösungen. Dazu ruft die UL eini- } \\
\text { ge UT auf, ihre Lösungen vorzu- } \\
\text { lesen. }\end{array}$ & & & \\
\hline $\begin{array}{l}\text { Festigung } \\
12^{\circ}\end{array}$ & & $\begin{array}{l}\text { 2.6.: Detailliertes Lesen } \\
\text { Die UL erklärt Aufgabe } 4 \text { a) (Le- } \\
\text { sen Sie sich den folgenden Text } \\
\text { genau durch und beantworten Sie } \\
\text { die Fragen. Prïfen Sie Ihre An- } \\
\text { tworten mit Ihrem } \\
\text { Banknachbarn). Die UL kontrol- } \\
\text { liert die Antworten. }\end{array}$ & $\begin{array}{l}\text { Die UT lesen den Text in } \\
\text { Aufgabe 4. a) genau durch } \\
\text { und beantworten die dazu- } \\
\text { gehörigen Fragen, wobei sie } \\
\text { zuerst die Antworten mit ih- } \\
\text { rem Sitznachbarn über- } \\
\text { prüfen. }\end{array}$ & $\begin{array}{l}\text { a) Lesen, } \\
\text { Schreiben } \\
\text { b) Einzelarbe- } \\
\text { it, Partnerarbe- } \\
\text { it, Arbei- } \\
\text { tsblatt, Com- } \\
\text { puter }\end{array}$ & $\begin{array}{l}\text { Im Text werden } \\
\text { die Techniken } \\
\text { Input enhan- } \\
\text { cement durch } \\
\text { Farben und } \\
\text { Inputflut } \\
\text { verwendet. }\end{array}$ \\
\hline
\end{tabular}

\section{SCHLUSSTEIL}

\begin{tabular}{|c|c|c|c|c|}
\hline $\begin{array}{l}\text { Phasen der } \\
\text { Stoffbearb } \\
\text { eitung/Zeit }\end{array}$ & Ziele & Aktivitäten der Untersuchungsleiterin & $\begin{array}{l}\text { Aktivitäten der Untersuch- } \\
\text { ungsteilnehmer }\end{array}$ & $\begin{array}{l}\text { a) Arbeits- } \\
\text { formen } \\
\text { b) Sozialformen, } \\
\text { Medien und Ma- } \\
\text { terialien }\end{array}$ \\
\hline $\begin{array}{l}\text { Anwen- } \\
\text { dung/ } \\
\text { Transfer } \\
25^{\circ}\end{array}$ & $\begin{array}{l}\text { Die UT sol- } \\
\text { len mithilfe } \\
\text { des vorgege- } \\
\text { ben Materi- } \\
\text { als einen } \\
\text { Text über ih- } \\
\text { ren Alltag als } \\
\text { Schulkinder } \\
\text { produzieren. }\end{array}$ & $\begin{array}{l}\text { 3.: Mein Schulalltag als Kind } \\
\text { Die UL erklärt die letzte Aufgabe auf dem Ar- } \\
\text { beitsblatt (Machen Sie eine Zeitreise in ihre } \\
\text { Kindheit. Verfassen Sie einen ähnlichen } \\
\text { Abschnitt uiber Ihren Alltag als Schulkinder. } \\
\text { Benutzen Sie dabei möglichst die folgenden } \\
\text { Angaben, aber ergänzen Sie diese auch mit ei- } \\
\text { genen Angaben). Die UL achtet auf die A- } \\
\text { usführung der Aufgabe und überprüft mit den } \\
\text { UT die Lösungen. Die UL achtet darauf, dass } \\
\text { alle Varianten, die von den UT selbst hinzu- } \\
\text { gefügt wurden, überprüft werden. }\end{array}$ & $\begin{array}{l}\text { Die UT verfassen einen } \\
\text { ähnlichen Abschnitt wie in Au- } \\
\text { fgabe 4. a) über ihren Alltag } \\
\text { als Schulkinder. Die UT be- } \\
\text { nutzen dabei möglichst die } \\
\text { vorgegebenen Angaben, aber } \\
\text { sie ergänzen diese auch mit ei- } \\
\text { nigen eigenen Beispielen. } \\
\text { Die UT antworten auf die } \\
\text { zusätzlichen Fragen der UL } \\
\text { und berichten ihren Alltag als } \\
\text { Kinder. }\end{array}$ & $\begin{array}{l}\text { a) Schreiben } \\
\text { b) Einzelarbeit, } \\
\text { Arbeitsblatt }\end{array}$ \\
\hline
\end{tabular}




\section{Anschrift der Autorin:}

Fakultät für Geistes- und Sozialwissenschaften in Osijek

Lorenz Jäger 9, Osijek

E-mail: isaricsokcevic@ffos.hr

\section{ODNOS JEZIČNE SVJESNOSTI I NAČINA POUČAVANJA U OVLADAVANJU GLAGOLI- MA KOJI (NE) MOGU TVORITI PASIV U NJEMAČKOM KAO STRANOM JEZIKU}

Glavni je cilj ovoga istraživanja rasvijetliti odnos svjesnosti, načina poučavanja te uspjeha u ovladavanju glagolima koji (ne) mogu tvoriti pasiv u njemačkome kao stranome jeziku. Istraživanja na području ovladavanja inim jezikom (Schmidt 1990; Robinson 1995; Leow 2001) potvrdila su Schmidtovu teoriju uočavanja (1990; 1995; 2001) koja pažnju drži ključnim elementom u učenju. Prema Schmidtu (isto) svi aspekti učenja jezika zahtijevaju određeni stupanj usredotočene pažnje, s time da različiti aspekti mogu zahtijevati više ili manje pažnje. Ovakvim se shvaćanjem procesa obrade jezičnoga unosa pozornost ne pridaje samo pažnji nego i svjesnosti. Važnu ulogu u ovome procesu imaju i razine svjesnosti. Schmidt (isto) uočava dvije razine svjesnosti: razinu uočavanja (engl. level of noticing) te razinu razumijevanja (engl. level of understanding). Polazi se od toga da se viša razina svjesnosti, razumijevanje određenog jezičnog unosa, može ostvariti na dva načina: eksplicitnim ili implicitnim učenjem i/ili poučavanjem (DeKeyser 2003; Ellis R. 2005). Kako bi se ove varijable što bolje istražile, provodio se eksperiment u prirodnim uvjetima u trima fazama. U istraživanju su sudjelovale dvije skupine sudionika: hrvatski srednjoškolski koji uče njemački kao strani jezik te studenti prve godine preddiplomskog studija njemačkoga jezika. Svaka od navedenih skupina dodatno je podijeljena u dvije podskupine te su izložene implicitnom ili eksplicitnom načinu poučavanja. Metodom testiranja prije izloženosti određenom načinu poučavanja, neposredno nakon i s dužim vremenskim odmakom (dva mjeseca) utvrdila se razina ovladanosti ciljnim skupinama glagola. Primjenjivala se i metoda glasnog navođenja misli kako bi se kodificirale razine svjesnosti. Prikupljeni podatci obrađeni su kvalitativno i kvantitativno statističkim postupkom analize varijance. Rezultati istraživanja upućuju na posebno pozitivno izraženu jaku interakciju između više razine svjesnosti, boljih rezultata na neposrednom testiranju i testiranju s odmakom te eksplicitnog načina poučavanja. Postoji i veza između niže razine svjesnosti te implicitnog načina poučavanja kod neposrednog testiranja što se nije moglo potvrditi na testiranju s vremenskim odmakom od dva mjeseca. Utvrđena je i interakcija između uspjeha sudionika koji se nalaze na višoj razini znanja (studenata) te niže razine svjesnosti, a ti isti ispitanici ostvaruju i bolji uspjeh na neposrednom testiranju. Dobiveni podaci potvrđuju Schmidtovu teoriju uočavanja (1990) te rezultate drugih sličnih empirijskih istraživanja (npr. Leow 1997, 2001, 2015; Medina 2015). 
Ivana Šarić Šokčević:

Die Beziehung zwischen der Bewusstheit und den Lehrmethoden beim Erlernen der (nicht) passivfähigen Verben im Deutschen als Fremdsprache

Ključne riječi: ovladavanje stranim jezikom; svjesnost; razine svjesnosti; eksplicitni i implicitni načini poučavanja. 